\title{
Anomalous Negative Fluorescence Anisotropy in Yellow Fluorescent Protein (YFP 10C): Quantitative Analysis of FRET in YFP Dimers
}

\author{
Xinghua Shi ${ }^{1}$, Jaswir Basran ${ }^{2}$, Harriet E. Seward ${ }^{2}$, William Childs ${ }^{1}$, Clive R. Bagshaw ${ }^{2}$, and \\ Steven G. Boxer ${ }^{1^{\star}}$ \\ 1Department of Chemistry, Stanford University, Stanford, CA 94305-5080, USA \\ 2Department of Biochemistry, University of Leicester, Leicester LE1 9HN, UK
}

\begin{abstract}
YFP is widely used as a genetically-encoded fluorescent marker in biology. In the course of a comprehensive study of this protein, we observed an unusual, negative fluorescence anisotropy at pH 6.0 (McAnaney, T. B., Zeng, W., Doe, C. F. E., Bhanji, N., Wakelin, S., Pearson, D. S., Abbyad, P., Shi, X. H., Boxer, S. G., and Bagshaw, C. R. (2005), Biochemistry 44, 5510-5524). Here we report that the fluorescence anisotropy of YFP 10C depends on protein concentration in the low micromolar range that was not expected. We propose that the negative anisotropy is a result of unidirectional Förster resonance energy transfer (FRET) in a dimer of YFP, with the donor chromophore in the neutral form and the acceptor chromophore in the anionic form. This unusual mechanism is supported by studies of a monomeric YFP (A206K YFP) and transient-absorption spectroscopy of YFP 10C. A detailed analysis of the chromophore transition dipole moment direction is presented. The anisotropy and rate constant of this energy transfer are consistent with values produced by an analysis of the dimer structure observed in crystals.
\end{abstract}

Green fluorescent protein (GFP) variants constitute a large family of valuable tools (1) that have revolutionized the field of biological imaging (2). Yellow fluorescent proteins (YFPs) that contain a $\mathrm{T} 203 \mathrm{Y}$ or $\mathrm{T} 203 \mathrm{~F}$ mutation are important members of this family, and they exhibit fluorescence emission red-shifted from that of wild-type GFP. A particular YFP construct, YFP 10C (EYFP), containing substitutions S65G/V68L/S72A/T203Y, has been widely used as a result of its commercial availability. The crystal structure of YFP $10 \mathrm{C}$ reveals a $\pi$-stacking interaction between the side-chain phenol of Tyr203 and the chromophore, and this feature has been proposed to be responsible for the spectral shifts in YFPs (3). YFPs also exhibit a tendency to dimerize at high concentrations with the dissociation constant, $K_{\mathrm{d}}$, reported to be on the order of $110 \mu \mathrm{M}$ at physiological $\mathrm{pH}$ (4). Zacharias et al. introduced an A206K mutation on the background of Q69K YFP 10C and discovered that this mutation, which is at the surface contact of crystallographic dimers of GFP (5) and YFP (3), increases the dissociation constant, $K_{\mathrm{d}}$, to a value on the order of $74 \mathrm{mM}(4)$, making this mutant essentially monomeric at $\mu \mathrm{M}$ concentrations and below.

The absorption spectrum of YFP 10C contains two bands that correspond to the neutral $\left(\lambda_{\max }=394 \mathrm{~nm}\right)$ and anionic $\left(\lambda_{\max }=514 \mathrm{~nm}\right)$ forms of the chromophore (Fig. 1A), with an

\footnotetext{
*E-mail: sboxer@ stanford.edu, Phone: (650)723-4482, Fax: (650)723-4817.

${ }^{\dagger}$ This work was supported in part by a grant from the NIH (GM27738 to S.G.B.) and the Leverhulme Trust (to C.R.B.). The fluorescence upconversion facilities are supported by the Medical Free Electron Laser Program of the Air Force Office of Scientific Research (Grant \#F49620-00-1-0349). X.S. acknowledges a William R. and Sara Hart Kimball Stanford Graduate Fellowship.
} 
apparent $\mathrm{p} K_{\mathrm{a}}$ of 6.3 in the presence of $44 \mathrm{mM}$ chloride (6). In the course of a comprehensive study of this protein (6), we observed that excitation of the neutral form at $\mathrm{pH} 6.0$ produces yellow fluorescence at $527 \mathrm{~nm}$, which exhibits a rapid decrease in anisotropy from an initial positive value to a negative value on the picosecond time scale (Fig. 1B). This unusual negative anisotropy is not observed for wild-type GFP (Fig. 1B). Fluorescence anisotropy is an intensity ratiometric measurement that describes the extent of polarization of emission upon excitation with polarized light. Anisotropy originates from the existence of transition moments of absorption and emission that lie in the chromophore structure along certain specific directions (7). Given the dependence of anisotropy on the angle between absorption and emission transition dipole moments (7), $\beta$, (see Fig. 1C):

$$
r_{0}(\beta)=\frac{3}{5} \cos ^{2} \beta-\frac{1}{5},
$$

Eq. 1

the decrease in YFP anisotropy to a negative value suggests a large change in the $\beta$ angle, approximately $62^{\circ}$, but the mechanism was unknown. Understanding how this apparent large reorientation of the transition dipole moment occurs in YFP may impact the design and improvement of FRET-based biosensors that involve YFP, since the efficiency of FRET depends on the transition moment orientation of the chromophores involved $(8,9)$ through the orientation factor (7), $\kappa^{2}$.

Several excited-state processes have been observed in fluorescent proteins. Our recent study of the excited-state dynamics in YFP 10C at pH 6.0 (6) suggested excited-state proton transfer (ESPT) as the pathway that produces the yellow-emitting, anionic form following the initial excitation of a neutral chromophore. This mechanism has been shown to occur in wild-type GFP (10) and several dual emission GFPs (deGFPs) (11-13). In the case of wild-type GFP, excitation of the neutral chromophore leads to excited-state proton transfer with a high quantum yield and creates the anionic form, which subsequently emits green fluorescence at $508 \mathrm{~nm}$ (Fig. 1D). ESPT in wild-type GFP does not significantly change the orientation of the emission transition dipole, as is reflected by the high anisotropy of the green fluorescence emitted by the deprotonated form following polarized excitation of the blue protonated form (Fig. 1B) (14). This high ESPT-associated anisotropy is in sharp contrast to the negative anisotropy observed for YFP (Fig. 1B); therefore, ESPT is unlikely to be the dominant mechanism in YFP for producing the anionic form following excitation of the neutral form.

Another process that could contribute to the negative fluorescence anisotropy in YFP is a cistrans isomerization of the chromophore. This reaction can twist the chromophore and thus lead to a reorientation of the transition dipole. Such a process has been well-documented in the literature for a variety of organic dyes, the visual pigment rhodopsin, photoactive yellow protein (15), and recently, a GFP-like protein named asFP595 (16), where a cis-trans isomerization is responsible for the chromophore's reversible photoswitching behavior. YFP exhibits a similar photoswitching behavior (6) and this may also be correlated with a cis-trans isomerization; however, the $\pi$-stacking between the side-chain phenol of Tyr203 and the chromophore observed in the crystal structure (3) can pose a substantial steric hindrance to a cis-trans isomerization of the chromophore, which may explain the relatively low quantum yield of reversible photoswitching when compared to fluorescence. Moreover, even if this reaction occurs, it may not lead to a change in the $\beta$ angle as large as $62^{\circ}$. Therefore, it seems unlikely that cis-trans isomerization is the origin of the negative anisotropy.

\section{Results and Discussion}

\section{Concentration Dependence in YFP 10C at pH 6.0}

At a concentration of $84.4 \mu \mathrm{M}$, time-resolved anisotropy of $527 \mathrm{~nm}$ fluorescence upon $400 \mathrm{~nm}$ excitation approximates what was reported previously (6): a rapid decrease to a negative value 
of -0.07 in less than $1 \mathrm{~ns}$, followed by a slower decay in absolute value on the ns time scale as a result of rotational diffusion (Fig. 2A). When diluted to $8.4 \mu \mathrm{M}$, YFP exhibits a noticeable change in anisotropy, approaching a higher value of -0.02 . Further dilution to $1.1 \mu \mathrm{M}$ leads to a positive anisotropy after the initial rapid decrease.

To examine if the anisotropy reaches higher values at lower concentrations, we used a steadystate spectrofluorimeter that allows for accurate characterization of fluorescence anisotropy at $\mathrm{nM}$ concentrations. Steady-state fluorescence anisotropy at $527 \mathrm{~nm}$ was measured for YFP excited at $400 \mathrm{~nm}$, with the concentration ranging from $20 \mu \mathrm{M}$ to $40 \mathrm{nM}$. As shown in Fig. 2B, the anisotropy increases with decreasing concentration. When YFP is diluted to $40 \mathrm{nM}$, the anisotropy reaches a value of 0.28 , close to the steady-state value observed for many fluorescent proteins. Note that this concentration dependence of anisotropy is not unique to YFP 10C; it is also observed for H148G YFP, a mutant of YFP 10C (see Fig. S1). This suggests that whatever process is responsible for the unusual anisotropy may be common among YFPs at certain $\mathrm{pH}$ and concentration conditions.

Several concentration-dependent processes can affect the observed fluorescence anisotropy. Trivial emission-reabsorption can depolarize the fluorescence and lead to a low anisotropy, but not a negative value. FRET can occur among identical chromophores in a well-defined system, such as a protein oligomer, and we will call this homo-FRET. As was demonstrated in an early study on chlorophyllide-substituted hemoglobin (17), fluorescence anisotropy decreases as a result of energy transfer unless the transition dipole moments happen to be parallel. In the case of two identical chromophores, the fluorescence should exhibit the following anisotropy

$$
r=\frac{k_{\mathrm{FRET}}^{\text {homo }} r_{\mathrm{FRET}}+\left(k_{f}+k_{\mathrm{FRET}}^{\mathrm{homo}}\right) r_{0}}{k_{f}+2 k_{\mathrm{FRET}}^{\text {homo }}},
$$

where $k_{\mathrm{FRET}}^{\text {homo }}$ and $k_{f}$ are the rate constants for homo-FRET and all the other decay processes, respectively; $r_{0}$ and $r_{F R E T}$ are the anisotropy values for the monomer and energy transfer in the dimer, respectively. According to Eq. 2, the overall anisotropy should be between $r_{0}\left(\right.$ when $\left.k_{f} \gg k_{\mathrm{FRET}}^{\text {homo }}\right)$ and $\left(r_{\mathrm{FRET}}+r_{0}\right) / 2$ (when $\left.k_{f} \ll k_{\mathrm{FRET}}^{\text {homo }}\right)$. The monomer anisotropy typically assumes a value between 0.3 and 0.4 , while the lowest value of FRET anisotropy is -0.2 ; thus, a negative anisotropy would not be expected for homo-FRET in a dimer with two identical chromophores. In fact, dimerization has been observed for GFP $(18)$ and YFP $(4,19)$ at high concentrations. Jung et al. reported a final anisotropy of $0.28 \pm 0.02$ following an ultrafast fluorescence depolarization in YFP upon direct excitation of the anionic chromophore, as a result of homo-FRET between two identical, anionic chromophores in a YFP dimer (20). Aside from emission-reabsorption and homo-FRET, other concentration-dependent processes may also exist and cannot be ruled out.

In addition to the unusual negative anisotropy observed here, the effect of protein concentration occurs in a region (Figs. 2A and B) that is much lower than expected. A previous study using analytical ultracentrifugation has shown that YFP dimerizes at $\mathrm{pH} 7.4$ with a $K_{\mathrm{d}}$ of $110 \mu \mathrm{M}$ (4) (we obtained a similar value of $100 \mu \mathrm{M}$ (19) at $\mathrm{pH} 7.5$ under our buffer conditions). Assuming this value for YFP 10C at pH 6.0 (see below and Appendix I), the monomer should have dominated the equilibrium at a total concentration of $8.4 \mu \mathrm{M}$. However, the anisotropy observed at this concentration still exhibits a rapid decrease to a negative value (Fig. 2A) and the transition in anisotropy from a negative to a positive value is evident at concentrations that are even lower (Fig. 2B). Therefore, whatever process is responsible for the unusual anisotropy (see below), the value of $K_{\mathrm{d}}$ at $\mathrm{pH} 6.0$ should be much lower than the aforementioned literature values obtained by analytical ultracentrifugation at physiological $\mathrm{pH}$ (see Concentration Dependence of Steady-State Excitation Spectra). 
In the experiments described here, the yellow fluorescence is observed from the anionic chromophore following excitation of the neutral form. Therefore, we look more closely at the protonation states involved for the chromophore at this $\mathrm{pH}$. When illuminated with $400 \mathrm{~nm}$ light, the neutral form $\left(\lambda_{\max }=394 \mathrm{~nm}\right)$ is excited much more than the anionic form $\left(\lambda_{\max }=\right.$ $514 \mathrm{~nm}$ ), as a result of the at least 20 -fold difference in molar extinction coefficient. This is confirmed by comparing the excitation spectra for $527 \mathrm{~nm}$ fluorescence at $\mathrm{pH} 6.0$ and 9.0 (Fig. $\mathrm{S} 2 \mathrm{~A}$ ) which shows that following excitation at $400 \mathrm{~nm}$, most of the yellow fluorescence at $\mathrm{pH}$ 6.0 comes from excitation of the neutral form, rather than the high-energy tail of the anionic form's absorption band or the low-energy tail of the near-UV band. As has been shown previously (6), the neutral YFP chromophore fluoresces blue $\left(\lambda_{\max }=465 \mathrm{~nm}\right)$, thus, it can serve as an energy-transfer donor to an anionic chromophore, which absorbs between 400 and $550 \mathrm{~nm}$ and subsequently fluoresces yellow $\left(\lambda_{\max }=527 \mathrm{~nm}\right)$. Besides sufficient spectral overlap for FRET between the neutral and anionic chromophores, the relatively large molar extinction coefficient for the latter $(21)\left(\varepsilon_{514}=83400 \mathrm{M}^{-1} \mathrm{~cm}^{-1}\right)$ can make it a good antenna for harvesting energy from the former when they are located in close proximity. Conversely, emission from the anionic form has very poor overlap with absorption of the neutral form. Therefore, in a YFP dimer that has one chromophore in the neutral form and the other in the anionic form, excitation can migrate only from the neutral donor to the anionic acceptor chromophore in a unidirectional fashion, while the FRET in the reverse direction has a very low probability. Since two chromophores with distinct characteristics are involved here, we will call this hetero-FRET.

If hetero-FRET is the dominant process at high concentrations that leads to the yellow fluorescence from an anionic chromophore following excitation of the neutral form, this process should have a rate constant much greater than that of ESPT, i.e. $k_{\mathrm{FRET}}^{\text {hetero }} \gg k_{\mathrm{ESPT}}$. Otherwise ESPT would dominate, leading to the rapid formation of the anionic state on the donor and a positive fluorescence anisotropy. FRET in a YFP hetero-dimer is unidirectional so the final anisotropy reflects $r_{F R E T}$ only, rather than the average of $r_{F R E T}$ and $r_{0}$ as is the case for a rapid, bidirectional homo-FRET. Thus, if a large angle exists between the two transition dipole moments, a negative value can be observed for the final anisotropy in a unidirectional hetero-FRET but not in a homo-FRET.

\section{Concentration Dependence of Steady-State Excitation Spectra}

Hetero-FRET in YFP 10C at pH 6.0 can also explain the concentration dependence of fluorescence excitation spectra of this protein. The excitation spectra for $527 \mathrm{~nm}$ fluorescence shown in Fig. 2C are normalized to the intensity at $450 \mathrm{~nm}$ instead of $514 \mathrm{~nm}$, the peak position of the anionic form, so that the region around $394 \mathrm{~nm}$ is more visible. As the YFP concentration is lowered, the excitation efficiency at $394 \mathrm{~nm}$ is decreased dramatically (Fig. 2C). This concentration dependence in excitation spectra is the opposite of the trend seen in absorption spectra (Fig. 2D). Thus, the quantum yield for $527 \mathrm{~nm}$ fluorescence following excitation of the neutral chromophore is much greater at higher concentrations than at lower concentrations. This observation is indicative of a channel that is present only in the high-concentration form of YFP; unidirectional FRET is consistent with such a channel, since it exists only in a YFP dimer, but not in a monomer. On the other hand, the concentration dependence of the quantum yield from exciting the neutral form is not predicted by other mechanisms that can produce the yellow fluorescence, including ESPT and direct excitation of the anionic form at $394 \mathrm{~nm}$. Therefore, these mechanisms are not as efficient as the unidirectional hetero-FRET in YFP $10 \mathrm{C}$ at $\mathrm{pH}$ 6.0. A more quantitative analysis of the various competing processes, including FRET, ESPT and all the other channels, is provided below. This concentration dependence of the excitation spectra was also used to obtain a value of $10 \sim 15 \mu \mathrm{M}$ for $K_{\mathrm{d}}$, as shown in Appendix I. Note that the YFP concentration corresponding to a 50\% change in anisotropy (Fig. 2B) cannot be used directly as a measure of $K_{\mathrm{d}}$, because of the different fluorescence 
quantum yields of the low- and high-anisotropy forms. After a correction for this difference, the value of $K_{\mathrm{d}}$ is in reasonable agreement with that obtained from the excitation spectra.

\section{Monomeric YFP}

The unidirectional hetero-FRET discussed thus far is proposed to result from the formation of a YFP dimer at $\mathrm{pH}$ 6.0, with one YFP chromophore in a neutral form and the other an anionic form. In addition to this neutral-anionic type of dimer, other combinations, i.e. neutral-neutral and anionic-anionic, certainly exist in YFP at $\mathrm{pH} 6.0$ given the apparent $\mathrm{p} K_{\mathrm{a}}$ of 6.3 for the chromophore; however, the contribution of these species to the yellow fluorescence with 394 $\mathrm{nm}$ excitation is very small due to the relatively poor quantum yield from ESPT (see the quantitative analysis of competing processes below) and low direct excitation efficiency for the anionic chromophore at $394 \mathrm{~nm}$.

The importance of dimerization is supported by the data for a monomeric YFP, the A206K variant (4) of YFP 10C at $\mathrm{pH}$ 6.0. The absorption spectrum of A206K YFP at $\mathrm{pH} 6.0$ is essentially the same as YFP 10C, with a similar apparent $\mathrm{p} K_{\mathrm{a}}$ for the chromophore (Fig. 3A). As Fig. 3B shows, for $527 \mathrm{~nm}$ fluorescence low excitation efficiency is observed for the neutral chromophore at $400 \mathrm{~nm}$, and this is consistent with the absence of the FRET mechanism in this monomeric protein. The excitation spectrum of A206K YFP is comparable to that of YFP $10 \mathrm{C}$ at low concentrations (Fig. 2C). In both cases, only ESPT and the possible direct excitation of other underlying bands at $400 \mathrm{~nm}$ are contributing to the fluorescence at $527 \mathrm{~nm}$; thus, a very low quantum yield is observed when exciting at $400 \mathrm{~nm}$. The $527 \mathrm{~nm}$ fluorescence of A206K YFP at steady state exhibits a high anisotropy with $400 \mathrm{~nm}$ excitation (Fig. 3C), and this is in sharp contrast to the anisotropy of YFP 10C at similar concentrations (Fig. 2B and Fig. 3C). In addition, time-resolved fluorescence at $527 \mathrm{~nm}$ following $400 \mathrm{~nm}$ excitation also shows high and positive anisotropy for A206K YFP throughout the entire time window (Fig. 3D), which is consistent with the high anisotropy observed in steady-state experiments (Fig. $3 \mathrm{C})$.

The results for A206K YFP demonstrate that when dimerization is eliminated, following excitation of the neutral chromophore both the negative anisotropy and most of the yellow fluorescence observed in YFP 10C is gone. This supports the proposed mechanism for producing the negative anisotropy in YFP 10C as unidirectional hetero-FRET from a neutral donor chromophore to an anionic acceptor chromophore in a dimer. Other processes that also require dimerization can not be ruled out, including those involving an energy transfer from a higher excited state or a different conformation of the chromophore in one of the two protein molecules; however, we do not have evidence for the existence of these states. In particular, high anisotropy, i.e. close to 0.4 , is observed following excitation of YFP 10C at $400 \mathrm{~nm}$ for the $460 \mathrm{~nm}$ fluorescence over its short lifetime in upconversion experiments (6) and for the weak, blue emission band below $500 \mathrm{~nm}$ in steady-state experiments (data not shown). These observations are consistent with the donor state in the hetero-FRET being the neutral chromophore's first excited state, rather than a higher excited state or a different chromophore conformation, which may lead to a lower anisotropy for the donor as well.

Given the important role of YFP dimerization established here and previous suggestions that high salt conditions during crystallization seem to favor dimerization $(3,22)$, we examined the influence of increased ionic strength on spectroscopic observables (Figs. 3A-C). As these absorption, fluorescence excitation and anisotropy data indicate, increasing the $\mathrm{Na}_{2} \mathrm{SO}_{4}$ concentration in the $\mathrm{pH} 6.0$ buffer from 0 to $400 \mathrm{mM}$ has only minor and negligible effects on the spectra of YFP 10C and A206K YFP, respectively. This is not surprising, since it is likely that the hydrophobic residues at the dimer interface are mostly responsible for the dimerization in YFP 10C; therefore, a change in the bulk electrostatics does not significantly affect the dimerization. 


\section{Pump-Probe Spectra: ESPT versus FRET}

Unidirectional hetero-FRET in YFP dimers would not have appeared without the existence of two YFP populations, one with the chromophore in the neutral form and the other anionic. Another process, ESPT, was originally considered as the process that is responsible for the yellow fluorescence following $400 \mathrm{~nm}$ excitation of YFP 10C at pH 6.0 (6); however, the negative anisotropy clearly does not support this argument. ESPT and FRET have the similarity that both are associated with a large shift in energy from absorption to emission. However, a key distinction exists between these two processes in terms of the number of chromophores involved, aside from the fortuitous difference in anisotropy (fortuitous in the sense that this only occurs for certain orientations of the transition moments (see below)). For ESPT, only one chromophore is involved (Fig. 4A). Initially, the neutral form, called A (10), occupies the ground state (left panel). After absorbing a blue photon, it is raised to the first excited state, $\mathrm{A}^{*}$, and this leads to a bleach in the ground-state absorption of the neutral form (middle panel). Subsequently, ESPT deprotonates the neutral form and the anionic form, called I*, is created (right panel) and the ground-state bleach of the neutral form is not recovered at this moment. For FRET, two chromophores are involved (Fig. 4B). Initially, the donor chromophore 1 exists in the neutral form and the acceptor chromophore 2 in the anionic form on the ground state (left panel). Note that these notations are used here instead of the conventional language for FRET, i.e. $\mathrm{D}$ and $\mathrm{A}$ for the donor and acceptor, respectively, to avoid the confusion with the A form of the chromophore described above. After absorbing a blue photon, chromophore 1 is raised to its first excited state, $1^{*}$, and this leads to a bleach in the ground-state absorption (middle panel). When FRET occurs between $1 *$ and 2, 1* is deexcited and 2 is simultaneously raised to its first excited state, $2^{*}$ (right panel). As a result, the ground-state bleach of chromophore 1 is now recovered while a new bleach band should appear for chromophore 2 .

We obtained the transient-absorption spectra for both wild-type GFP and YFP 10C at 450 picoseconds, following excitation of the neutral chromophore with femtosecond pulses at 390 $\mathrm{nm}$. In pump-probe experiments, both bleach in the ground-state absorption and stimulated emission from the excited state lead to more light in the probe beam reaching the detector with the pump on than off; therefore, they can both give rise to a negative transient-absorption feature. In contrast, absorption from a state transiently present leads to less light in the probe beam reaching the detector with the pump on than off; therefore, this can result in a positive transient-absorption feature. For GFP (Fig. 4C), the ground-state bleach of the neutral form is clearly visible as a negative band near $400 \mathrm{~nm}$ and no such bleach is observed for the anionic form at $475 \mathrm{~nm}$. These features agree well with those reported previously (23), where a more complete bandshape was shown for the neutral form including the region below $400 \mathrm{~nm}$ that is beyond the spectral window of our instrument. Therefore, the transient-absorption spectrum of GFP at $\sim 450$ picoseconds is consistent with the ESPT scenario shown in Fig. 4A (right panel). For YFP at $\mathrm{pH}$ 6.0, in contrast, we found no evidence for a ground-state bleach of the neutral chromophore (Fig. 4D), and this is clearly different from the situation shown for GFP in Fig. 4C. Furthermore, a negative feature at $\sim 480 \mathrm{~nm}$ is well resolved in the $\Delta \mathrm{A}$ spectrum (Fig. 4D), and this feature is likely due to the vibronic shoulder of bleached ground-state absorption of the anionic chromophore (see Fig. 1A). Stimulated emission from the anionic chromophore and absorption of excited state(s) can contribute to the signal detected at this wavelength; however, the former should have little intensity below $500 \mathrm{~nm}$ (Fig. 1A), and the latter has a positive sign for $\Delta \mathrm{A}$. Thus, these two should not contribute to the negative $\Delta \mathrm{A}$ at $\sim 480 \mathrm{~nm}$, so we assigned the negative feature at $\sim 480 \mathrm{~nm}$ to the ground-state bleach of the anionic chromophore. We were unable to resolve a bleach band with a maximum at $514 \mathrm{~nm}$ in Fig. 4D, which would be expected for the anionic form; however, it is likely that this is due to spectral overlap with the stimulated emission peaked at $527 \mathrm{~nm}$ due to the small Stokes shift, as illustrated in Fig. 4E. Therefore, both the absence of ground-state bleach for the neutral 
chromophore and the presence of such bleach for the anionic chromophore are consistent with the FRET scenario shown in Fig. 4B (right panel).

The pump-probe data are also in good agreement with the argument that instead of ESPT, hetero-FRET is the dominant process producing the anionic, excited state in YFP 10C at pH 6.0 following excitation of the neutral form at high concentrations. If $k_{\mathrm{ESPT}} \gg k_{\mathrm{FRET}}^{\text {hetero }}$, features similar to those seen in Fig. 4C would be expected for YFP. The transient-absorption experiment described here provides a useful way to differentiate FRET from ESPT (Figs. 4A versus $4 \mathrm{~B}$ ), and this approach should also be applicable even in a case without the fortuitous negative anisotropy.

\section{Kinetics of Competing Processes}

With steady-state and time-resolved fluorescence experiments on YFP 10C and A206K YFP and pump-probe experiments on YFP 10C and GFP, we have demonstrated qualitatively that hetero-FRET, instead of ESPT, is the dominant pathway producing the anionic form of the YFP chromophore in the excited state, following excitation of the neutral form in YFP 10C at high concentrations. In the following we compare the kinetics of the relevant competing processes in a more quantitative way.

As shown in the minimal model, Scheme 1, following excitation of the neutral form, $\mathrm{YFPH}_{\mathrm{D}}$, the excited state, $\mathrm{YFPH}_{\mathrm{D}}$, is first created and subsequently undergoes radiative decay, hetero-FRET, ESPT and other nonradiative decays with the rate constants $k_{r, 1}$,

$k_{\mathrm{FRET}}^{\text {hetero }}, k_{E S P T}$ and $k_{n, 1}$, respectively. Hetero-FRET and ESPT are assumed to be the only two processes following excitation of $\mathrm{YFPH}_{\mathrm{D}}$ that can produce the anionic form in the excited state, $\mathrm{YFP}^{-*}$, which can be either $\mathrm{YFP}_{\mathrm{A}}{ }^{-*}$ or $\mathrm{YFP}_{\mathrm{D}}{ }^{-*}$. They subsequently undergo radiative and nonradiative decays with the rate constants $k_{r, 2}$ and $k_{n r}, 2$, respectively. In addition, the connection between $\mathrm{YFPH}_{\mathrm{D}}$ and $\mathrm{YFP}_{\mathrm{D}}{ }^{-}$in the ground state is relatively slow; this has been investigated previously (6) with stopped-flow and pressure-jump techniques.

The expressions for the quantum yields of yellow fluorescence from $\mathrm{YFP}^{-*}$ following excitation of $\mathrm{YFPH}_{\mathrm{D}}$ in the absence and presence of hetero-FRET can be derived as

$$
\begin{gathered}
\Phi_{Y F P^{*}}=\frac{k_{r, 2}}{k_{r, 2}+k_{n r, 2}} \cdot \frac{k_{\mathrm{ESPT}}}{k_{r, 1}+k_{n r, 1}+k_{\mathrm{ESPT}}}, \\
\Phi_{Y F-^{*} *}=\frac{k_{r, 2}}{k_{r, 2}+k_{n r, 2}} \cdot \frac{k_{\mathrm{FRET}}^{\text {hetero }}+k_{\mathrm{ESPT}}}{k_{r, 1}+k_{n r, 1}+k_{\mathrm{FRET}}^{\text {hetero }}+k_{\mathrm{ESPT}}},
\end{gathered}
$$

respectively. For YFP 10C at low concentrations (Fig. 2C, blue curve) and the monomeric YFP (Fig. 3B, red curve), Eq. 3 can be applied because hetero-FRET is absent. In Eq. 3, the first term in the product on the right side can be assumed to equal the quantum yield of the directlyexcited anionic form. Thus, we divide the intensity at $394 \mathrm{~nm}$ by that at $450 \mathrm{~nm}$ and correct it for $1-10^{-\mathrm{A} \lambda} 1$ at these two wavelengths, and the result should equal the second term in the product on the right side of Eq. 3. Using the data from A206K YFP (Fig. 3B, red curve), we obtained $k_{E S P T} /\left(k_{r, 1}+k_{n r, 1}+k_{E S P T}\right)=0.0058$ and thus, $k_{E S P T} /\left(k_{r, 1}+k_{n r, 1}\right)=0.0058$. Similarly, using Eq. 4 and the data for YFP 10C at high concentrations (Fig. 2C, red curve), we obtained $\left(k_{\mathrm{FRET}}^{\text {hetero }}+k_{\mathrm{ESPT}}\right) /\left(k_{r, 1}+k_{n r, 1}+k_{\mathrm{FRET}}^{\text {hetero }}+k_{\mathrm{ESPT}}\right)=0.045$ and $k_{\mathrm{FRET}}^{\text {hetero }} /\left(k_{r, 1}+k_{n r, 1}\right)=0.041$. As a result, $k_{\mathrm{FRET}}^{\text {hetero }} / k_{\mathrm{ESPT}}=7$, and this supports the previous conclusion that hetero-FRET is the dominant channel producing $\mathrm{YFP}^{-*}$ following excitation of $\mathrm{YFPH}_{\mathrm{D}}$ at high concentrations. Based on the average lifetime of $\mathrm{YFPH}_{\mathrm{D}}{ }^{*}, 1.4 \pm 0.1 \mathrm{ps}$, as previously measured by upconversion experiments (6), we obtain $k_{\text {FRET }}^{\text {hetero }}=(2.8 \pm 0.2) \times 10^{10} \mathrm{~s}^{-1}$. 
Note that the ratio $k_{E S P T} /\left(k_{r, 1}+k_{n r, 1}\right)$ above has been overestimated because the contribution from the direct excitation of $\mathrm{YFP}^{-}$is relatively significant at $394 \mathrm{~nm}$. At the same time, the

ratio $k_{\mathrm{FRET}}^{\text {hetero }} /\left(k_{r, 1}+k_{n r, 1}\right)$ has been underestimated because even at high concentrations of YFP $10 \mathrm{C}$ there still exists a large percentage of neutral-neutral dimer, which contributes also to the absorption at $394 \mathrm{~nm}$, but little fluorescence at $527 \mathrm{~nm}$, due to the slow ESPT rate. Therefore, $k_{\mathrm{FRET}}^{\text {hetero }} / k_{\mathrm{ESPT}}$ should be even greater than the aforementioned value, 7. After the percentage of neutral-neutral dimer is accounted for, we obtain the corrected $k_{\mathrm{FRET}}^{\text {hetero }}=(8.1 \pm 0.6) \times 10^{10} \mathrm{~s}^{-1}$.

\section{Structural Analysis Requirements}

Given that at high concentrations a negative value is observed for the final anisotropy (Fig. 1B), a large angle must exist between the absorption dipole of the donor, neutral chromophore and the emission dipole of the acceptor, anionic chromophore in the YFP dimer (Eq. 1 and Fig. 1C). Although no information is available on the structure(s) of the dominant dimeric species in solution, the X-ray crystal structure of YFP contains a dimer as shown in Fig. 5 (PDB code: 1YFP (3)). We believe this is relevant, since a disruption at the dimer interface by mutation A206K that was based on this structure makes YFP monomeric. Therefore, the dimer structure in crystals is considered a reasonable model for the solution structure, and it allows us to analyze the structural basis of the transfer angle and estimate the rate constant of unidirectional FRET from the relevant distance and orientations. In order to perform a quantitative analysis we need to know the transition dipole moment orientation of the chromophore in the framework of the molecular axes. Although this is not directly available in literature for YFP, recent studies on wild-type GFP (24-27) provide an opportunity to obtain this information for the GFP chromophore which is a reasonable model for the YFP chromophore, given their similar chemical structures except the $\pi$-stacking observed only for YFP (3).

\section{Transition Moment Orientation}

Previously, polarized absorption spectra of orthorhombic GFP crystals with $P_{212121}$ symmetry were measured to obtain the direction of electronic absorption moment of the chromophore relative to the molecular axes of the protein (28). The analysis presented there (28) has flaws and a correction is provided in Appendix II. Recently, time-resolved mid-infrared (IR) experiments on wild-type GFP using the visible pump/IR probe technique (24-27) have provided new opportunities to obtain information on the transition moment orientation in the context of the orientations of local IR oscillator directions. As demonstrated by Stoner-Ma et al. $(26,27)$ and van Thor et al. $(24,25)$, these experiments yield valuable information regarding the changes to vibrational modes in the chromophore and surrounding protein matrix following optical excitation of the neutral chromophore. This information has been useful in delineating the key structural events relevant to the ESPT in GFP, in particular the protonation of E222 (24-27). As detailed in these references, the transient absorption at $1712 \mathrm{~cm}^{-1}$ that grows on the picosecond time scale results from the carbonyl stretch of protonated carboxylic acid in E222 and this mode is named $\mathrm{C}=\mathrm{O}(222)$. In addition, many other modes have also been revealed from the transient IR experiments, including the instantaneous bleach well resolved at 1681 $\mathrm{cm}^{-1}$ that exhibits no further evolution over 200 picoseconds (24-27). This band corresponds to the carbonyl stretch of the neutral chromophore in the electronic ground state (see references 24-27) and is named $\mathrm{C}=\mathrm{O}(\mathrm{Cro})$. Furthermore, Stoner-Ma et al. recently performed isotopic substitutions in the chromophore with ${ }^{13} \mathrm{C}$ at $\mathrm{C} 2$ and $\mathrm{CB} 2$ positions (Fig. 6) by labeling GFP with 1 - and $3-{ }^{13} \mathrm{C}$ tyrosine, respectively (26); the data provide strong support for this mode being localized mostly along $\mathrm{C} 2=\mathrm{O} 2$ (Fig. 6), which is also consistent with the previous assignment made for a synthetic model chromophore 4'-hydroxybenzylidene-2,3-dimethylimidazolinone (HBDI) (29). It is important for the following analysis that $\mathrm{C}=\mathrm{O}(\mathrm{Cro})$ and $\mathrm{C}=\mathrm{O}$ (222) are carbonyl-localized modes, that is, their vibrational transition moments are parallel to the $\mathrm{C}=\mathrm{O}$ bond axes, such that their anisotropy values measured by the polarization-resolved, 
visible pump/IR probe experiment (26) can be utilized to obtain the projection of the electronic transition moment through Eq. 1, given the well-defined directions of these bond vectors in the molecular axis system. Note that Stoner-Ma et al. did not present such an analysis (26); therefore, we performed this analysis using their vibrational dichroism results.

As presented in Appendix II, the orientation of $\mathrm{C}=\mathrm{O}(222)$ relative to the electronic transition moment of the chromophore is less certain than that of $\mathrm{C}=\mathrm{O}(\mathrm{Cro})$, because of the greater structural disorder of the E222 side chain and the possibility of a change in its orientation upon optical excitation and subsequent ESPT. With the assumption that the electronic transition moment of GFP lies in the chromophore plane defined by 3 noncollinear atoms, OH, $\mathrm{O} 2$ and N2 (Fig. 6), we constructed a vector representing the orientation of electronic transition moment that is rotated from the vector $\mathrm{OH} \rightarrow \mathrm{O} 2$ by an angle $\alpha$ towards the vector $\mathrm{OH} \rightarrow \mathrm{N} 2$ (Fig. 6). In this definition, $\alpha$ can range from -180 to $180^{\circ}$; however, the region from -90 to $90^{\circ}$ contains all the unique line directions. Thus, only this region is considered. The sign of $\alpha$ corresponds to the direction of rotation, positive for clockwise and negative for counterclockwise. As shown in Figs. 7A and B and Appendix II, the vibrational dichroism data by Stoner-Ma et al. (26) were used to obtain a value of $6.5 \pm 5^{\circ}$ for $\alpha$ that defines the absorption moment orientation in the plane of the neutral GFP chromophore (Fig. 6).

\section{Structural Analysis of Unidirectional FRET}

To utilize this information about the transition dipole orientation in GFP to evaluate the fluorescence anisotropy associated with unidirectional FRET in the YFP hetero-dimer, we make a number of simplifying assumptions: $(i)$ The available structure of YFP dimer in crystals (Fig. 5, PDB code: 1YFP (3)) represents the structure in solution. This is consistent with the fact that the YFP dimerization tendency is essentially eliminated by mutation A206K (4) (Fig. 3 ), which is at the surface contact of a crystallographic YFP dimer (3). Furthermore, mixtures of YFP 10C and excessive A206K YFP at pH 6.0 exhibited intermediate values of anisotropy (data not shown) that are consistent with the YFP 10C molecules forming independent faceto-face dimers at A206, with no indication of a dimer being formed between the A206 face of a YFP 10C molecule and another hydrophobic patch on a A206K YFP molecule. (ii) The absorption dipole orientation obtained as described above for the neutral chromophore in GFP applies to YFP as well. This is reasonable given the close similarity in the chemical structures of the chromophore, despite the $\pi$-stacking feature observed for YFP (3) only. It is not likely that this feature would appreciably affect the transition dipole orientation, but this could be tested by polarization-resolved, visible pump/IR probe measurements on YFP. (iii) For the neutral chromophore of YFP, the orientation of the absorption transition dipole approximates that of the emission dipole. This is supported by the high anisotropy, $0.38 \pm 0.03$, observed for the $460 \mathrm{~nm}$ fluorescence from the neutral YFP chromophore (6). In addition, such an approximation can also be made for the anionic chromophore. (iv) For a YFP chromophore, the transition dipole orientation of the neutral form approximates that of the anionic form. This is supported by the observation for GFP that upon excitation of the neutral form, high anisotropy is observed for the green fluorescence from the anionic form (14) (Fig. 1B). Note that this assumption can also be tested by polarization-resolved, visible pump/IR probe experiments.

With these assumptions, we calculated the FRET anisotropy in the YFP hetero-dimer as a function of angle $\alpha$ (Fig. 7C, green curve). For $\alpha=6.5 \pm 5^{\circ}$, the anisotropy falls within the range from -0.17 to -0.06 , with the boundaries corresponding to the $\alpha$ values that are one standard deviation away from the center. In addition, we also considered two scenarios with the transition dipole of the acceptor, anionic chromophore being rotated away from the orientation specified by the above $\alpha$ angle clockwise or counterclockwise by $15^{\circ}$ (Fig. 7C, red and blue curves). Note that this angle of $15^{\circ}$ is provided here only to examine the sensitivity 
of this anisotropy analysis. For these two scenarios, the corresponding anisotropy ranges are $(-0.2,-0.15)$, and $(-0.09,0.05)$, respectively. Besides the possible deviation in the transition dipole of the acceptor considered above, it is also conceivable that YFP's transition dipole may not be rigorously in the chromophore plane. For this reason, we examined two more scenarios with the transition dipole being rotated out of the plane by 15 or $-15^{\circ}$, where the positive sign corresponds to the out-of-plane component pointing from the plane of the paper (Fig. 6) towards the reader and a negative sign pointing away from the reader. To simplify the discussion, we let the in-plane component be defined by the same angle of $\alpha=6.5^{\circ}$ for both the donor and the acceptor and obtained 0.05 and -0.19 for the anisotropy in the two scenarios, respectively. These scenarios all produce a low or negative value for the FRET anisotropy in a YFP hetero-dimer, consistent with the experimental fluorescence anisotropy measured for YFP 10C at high concentrations (Fig. 1B). Therefore, this analysis is consistent with the crystal structure of YFP dimer being a good model for the solution structure, and is consistent with the unidirectional FRET being responsible for the anomalous negative anisotropy in YFP 10C at pH 6.0.

We also estimated the rate constant of unidirectional FRET in a YFP hetero-dimer based on the Förster dipole-dipole energy transfer theory (30), in addition to the structural information described above and the spectroscopic parameters discussed earlier. The prediction of the Förster theory can be expressed in a convenient form

$$
k_{\mathrm{FRET}}^{\text {hetero }}=\frac{R_{0}^{6}}{R^{6}} k_{f, 1},
$$

where $k_{\mathrm{FRET}}^{\text {hetero }}$ is the first-order, energy-transfer rate constant, $R_{0}$ is the Förster critical distance that is characteristic of the chromophore pair, $R$ is the interchromophore distance, and $k_{f, 1}$ is the excited-state decay rate observed for the donor in the absence of the acceptor. The Förster critical distance $R_{0}$ is given by (7)

$$
R_{0}^{6}=\frac{9000(\ln 10) \kappa^{2} \Phi_{1 *} J_{\lambda}}{128 \pi^{5} N_{A} n^{4}}
$$

where $\kappa^{2}$ is the orientation factor that describes the relative orientation of the transition dipoles of the donor and acceptor, $\Phi_{1 *}$ is the fluorescence quantum yield of the donor in the absence of the acceptor, $J_{\lambda}$ is the overlap integral depicting the degree of spectral overlap between the donor emission and acceptor absorption, $N_{A}$ is Avogadro's number, and $n$ is the refractive index of the medium.

As detailed in Appendix III, we obtained the parameters in Eqs. 5 and 6 (see Fig. 7D for the orientation factor $\kappa^{2}$ ) and thus, the rate constant for FRET in YFP hetero-dimer,

$k_{\mathrm{FRET}}^{\text {hetero }}($ calc $)=8.4 \times 10^{10} \mathrm{~s}^{-1}$. Note that the largest source of error in $k_{\mathrm{FRET}}^{\text {hetero }}$ (calc) is likely to be the distance $R$ that is not well defined as a result of the sixth power dependence in Eq. 5. That

is, even a small error in $R, e . g .5 \%$, can lead to a rather large deviation in $k_{\mathrm{FRET}}^{\text {hetero }}$ (calc) on the order of $30 \%$. Nevertheless, the value produced by the prediction of the Förster theory is in good agreement with the experimental value we obtained earlier, $(8.1 \pm 0.6) \times 10^{10} \mathrm{~s}^{-1}$, from fluorescence quantum yield measurements (see Kinetics of Competing Processes). This agreement further supports our finding of the unidirectional FRET in a hetero-dimer of YFP $10 \mathrm{C}$ at $\mathrm{pH} 6.0$ and validates the analyses we performed above for this process. We emphasize that the YFP hetero-dimer studied here provides one of the few FRET systems based on fluorescent proteins that can be studied in detail, as structural models are often unavailable in fusion pairs of fluorescent proteins $(9,31)$ and a full analysis of the transition moment direction has not been available before. The oligomerization state (dimer) present in this minimal system 
also allows for a straightforward assignment of the donor and acceptor chromophores, which is often unavailable in fluorescent protein systems involving higher oligomer states $(32,33)$.

\section{Potential Biological Applications}

The concentration dependence of the YFP anisotropy might be useful in some biological applications, e.g. the proteomic analysis of individual living cells. This type of analysis has become a valuable tool for quantitatively assessing cellular behavior (34-38). Since YFP 10C is often fused genetically to a target protein, one could measure the concentration of the target protein conveniently by monitoring the YFP or H148G YFP anisotropy with sensitive fluorescence techniques (Fig. 2B). This method can be attractive compared to other fluorescence methods, because fluorescence anisotropy does not require a nontrivial intensity calibration, as in other methods that depend on measuring the absolute fluorescence intensity $(34,35)$. Therefore this approach may provide a convenient way of specifically characterizing protein abundance in single living cells in situ, without the need to lyse the cell (38). In addition, the temporal information yielded by this real-time approach can also provide further details of cellular dynamics and thus be useful to cell biology research. With a careful design of the linker region, the impact of the target protein on the anisotropy of YFP tag may be minimized.

Remaining challenges may include the interference of intracellular autofluorescence, the concentration tag's applicable $\mathrm{pH}$ range, and the effective concentration range. Periasamy et al. demonstrated that advanced imaging techniques such as two-photon excitation microscopy can provide good spatial resolution and autofluorescence rejection (39). To explore the possibility of acquiring fluorescence anisotropy with two-photon excitation for YFP 10C at $\mathrm{pH} 6.0$, we collected time-resolved data at $527 \mathrm{~nm}$ using $5.7 \mathrm{~nJ}$, focused femtosecond $800 \mathrm{~nm}$ pulses (Fig. S3). The result clearly shows a negative anisotropy in YFP 10C at pH 6.0. Given the result we obtained for GFP under the same conditions (Fig. S3), we expect that a curve similar to the one shown in Fig. 2B can be found for YFP 10C, but with different asymptotic values. Wachter et al. determined the $\mathrm{p} K_{\mathrm{a}}$ for the chromophore in H148G YFP to be one $\mathrm{pH}$ unit higher than YFP 10C (3), making the former more suitable for potential applications at physiological $\mathrm{pH}$.

\section{Conclusion}

As the current study has demonstrated, the presences of protein dimerization, two protonation states and unidirectional FRET contribute together to the negative anisotropy observed in YFP 10C. Compared to the essentially negligible ESPT, FRET is much faster, but it is still slower than radiative and nonradiative decays of the neutral form in the excited state. Therefore, appreciable amount of yellow fluorescence is observed for YFP 10C at high concentrations through the FRET mechanism following excitation of the neutral chromophore, but the quantum yield is still about 20 -fold lower than direct excitation of the anionic chromophore.

\section{Supplementary Material}

Refer to Web version on PubMed Central for supplementary material.

\section{Abbreviations}

YFP, yellow fluorescent protein; FRET, Förster resonance energy transfer; GFP, green fluorescent protein; ESPT, excited-state proton transfer; IR, infrared; HBDI, 4'hydroxybenzylidene-2,3-dimethyl-imidazolinone.

\section{Acknowledgements}

We are very grateful to D. Ben Spry, Alexei Goun and Professor Michael D. Fayer for collecting the transientabsorption data, Karen Kallio and Professor S. James Remington at the University of Oregon for generously providing 
some of the YFP 10C and H148G YFP samples used in this study. We thank Ilya Finkelstein and Jayakumar Rajadas for useful discussions, Wei Zeng and Camille Doe for providing the YFP 10C sample used in initial experiments.

\section{References}

1. Shaner NC, Steinbach PA, Tsien RY. A guide to choosing fluorescent proteins. Nat. Methods 2005;2:905-909. [PubMed: 16299475]

2. Giepmans BNG, Adams SR, Ellisman MH, Tsien RY. Review - The fluorescent toolbox for assessing protein location and function. Science 2006;312:217-224. [PubMed: 16614209]

3. Wachter RM, Elsliger MA, Kallio K, Hanson GT, Remington SJ. Structural basis of spectral shifts in the yellow-emission variants of green fluorescent protein. Structure 1998;6:1267-1277. [PubMed: 9782051]

4. Zacharias DA, Violin JD, Newton AC, Tsien RY. Partitioning of lipid-modified monomeric GFPs into membrane microdomains of live cells. Science 2002;296:913-916. [PubMed: 11988576]

5. Yang F, Moss LG, Phillips GN. The molecular structure of green fluorescent protein. Nat. Biotechnol 1996;14:1246-1251. [PubMed: 9631087]

6. McAnaney TB, Zeng W, Doe CFE, Bhanji N, Wakelin S, Pearson DS, Abbyad P, Shi XH, Boxer SG, Bagshaw CR. Protonation, photobleaching, and photoactivation of yellow fluorescent protein (YFP 10C): A unifying mechanism. Biochemistry 2005;44:5510-5524. [PubMed: 15807545]

7. Lakowicz, JR. Principles of Fluorescence Spectroscopy. 3rd ed.. New York: Springer; 2006. p. 353-361.p. 443-451.

8. Nagai T, Yamada S, Tominaga T, Ichikawa M, Miyawaki A. Expanded dynamic range of fluorescent indicators for $\mathrm{Ca} 2+$ by circularly permuted yellow fluorescent proteins. Proc. Natl. Acad. Sci. U.S.A 2004;101:10554-10559. [PubMed: 15247428]

9. Shimozono S, Hosoi H, Mizuno H, Fukano T, Tahara T, Miyawaki A. Concatenation of cyan and yellow fluorescent proteins for efficient resonance energy transfer. Biochemistry 2006;45:6267-6271. [PubMed: 16700538]

10. Chattoraj M, King BA, Bublitz GU, Boxer SG. Ultra-fast excited state dynamics in green fluorescent protein: Multiple states and proton transfer. Proc. Natl. Acad. Sci. U.S.A 1996;93:8362-8367. [PubMed: 8710876]

11. Hanson GT, McAnaney TB, Park ES, Rendell ME, Yarbrough DK, Chu S, Xi L, Boxer SG, Montrose $\mathrm{MH}$, Remington SJ. Green fluorescent protein variants as ratiometric dual emission pH sensors. 1 . Structural characterization and preliminary application. Biochemistry 2002;41:15477-15488. [PubMed: 12501176]

12. McAnaney TB, Park ES, Hanson GT, Remington SJ, Boxer SG. Green fluorescent protein variants as ratiometric dual emission pH sensors. 2. Excited-state dynamics. Biochemistry 2002;41:1548915494. [PubMed: 12501177]

13. McAnaney TB, Shi X, Abbyad P, Jung H, Remington SJ, Boxer SG. Green fluorescent protein variants as ratiometric dual emission $\mathrm{pH}$ sensors. 3. Temperature dependence of proton transfer. Biochemistry 2005;44:8701-8711. [PubMed: 15952777]

14. Volkmer A, Subramaniam V, Birch DJS, Jovin TM. One- and two-photon excited fluorescence lifetimes and anisotropy decays of Green Fluorescent Proteins. Biophys. J 2000;78:1589-1598. [PubMed: 10692343]

15. Dugave C, Demange L. Cis-trans isomerization of organic molecules and biomolecules: Implications and applications. Chem. Rev 2003;103:2475-2532. [PubMed: 12848578]

16. Andresen M, Wahl MC, Stiel AC, Grater F, Schafer LV, Trowitzsch S, Weber G, Eggeling C, Grubmuller H, Hell SW, Jakobs S. Structure and mechanism of the reversible photoswitch of a fluorescent protein. Proc. Natl. Acad. Sci. U.S.A 2005;102:13070-13074. [PubMed: 16135569]

17. Kuki A, Boxer SG. Chlorophyllide-substituted hemoglobin tetramers and hybrids: preparation, characterization, and energy transfer. Biochemistry 1983;22:2923-2933. [PubMed: 6871173]

18. Wiehler J, Jung G, Seebacher C, Zumbusch Z, Steipe B. Mutagenic stabilization of the photocycle intermediate of green fluorescent protein (GFP). ChemBioChem 2003;4:1164-1171. [PubMed: 14613107] 
19. Zeng W, Seward HE, Malnasi-Csizmadia A, Wakelin S, Woolley RJ, Cheema GS, Basran J, Patel TR, Rowe AJ, Bagshaw CR. Resonance energy transfer between green fluorescent protein variants: complexities revealed with myosin fusion proteins. Biochemistry 2006;45:10482-10491. [PubMed: 16939200]

20. Jung G, Ma YZ, Prall BS, Fleming GR. Ultrafast fluorescence depolarisation in the yellow fluorescent protein due to its dimerisation. ChemPhysChem 2005;6:1628-1632. [PubMed: 16025560]

21. Cubitt AB, Woollenweber LA, Heim R. Understanding structure-function relationships in the Aequorea victoria green fluorescent protein. Methods Cell Biol 1999;58:19-30. [PubMed: 9891372]

22. Palm GJ, Zdanov A, Gaitanaris GA, Stauber R, Pavlakis GN, Wlodawer A. The structural basis for spectral variations in green fluorescent protein. Nat. Struct. Biol 1997;4:361-365. [PubMed: 9145105]

23. Kennis JT, Larsen DS, van Stokkum IH, Vengris M, van Thor JJ, van Grondelle R. Uncovering the hidden ground state of green fluorescent protein. Proc. Natl. Acad. Sci. U.S.A 2004;101:1798817993. [PubMed: 15608070]

24. van Thor JJ, Georgiev GY, Towrie M, Sage JT. Ultrafast and low barrier motions in the photoreactions of the green fluorescent protein. J. Biol. Chem 2005;280:33652-33659. [PubMed: 16033764]

25. van Thor JJ, Zanetti G, Ronayne KL, Towrie M. Structural events in the photocycle of green fluorescent protein. J. Phys. Chem. B 2005;109:16099-16108. [PubMed: 16853046]

26. Stoner-Ma D, Melief EH, Nappa J, Ronayne KL, Tonge PJ, Meech SR. Proton relay reaction in green fluorescent protein (GFP): Polarization-resolved ultrafast vibrational spectroscopy of isotopically edited GFP. J. Phys. Chem. B 2006;110:22009-22018. [PubMed: 17064171]

27. Stoner-Ma D, Jaye AA, Matousek P, Towrie M, Meech SR, Tonge PJ. Observation of excited-state proton transfer in green fluorescent protein using ultrafast vibrational spectroscopy. J. Am. Chem. Soc 2005;127:2864-2865. [PubMed: 15740117]

28. Rosell FI, Boxer SG. Polarized absorption spectra of green fluorescent protein single crystals: Transition dipole moment directions. Biochemistry 2003;42:177-183. [PubMed: 12515552]

29. He X, Bell AF, Tonge PJ. Isotopic labeling and normal-mode analysis of a model green fluorescent protein chromophore. J. Phys. Chem. B 2002;106:6056-6066.

30. Förster, Th. Zwischenmolekulare Energiewanderung Und Fluoreszenz. Ann. Phys 1948;2:55-75.

31. Fehr M, Frommer WB, Lalonde S. Visualization of maltose uptake in living yeast cells by fluorescent nanosensors. Proc. Natl. Acad. Sci. U.S.A 2002;99:9846-9851. [PubMed: 12097642]

32. Hosoi H, Mizuno H, Miyawaki A, Tahara T. Competition between energy and proton transfer in ultrafast excited-state dynamics of an oligomeric fluorescent protein red Kaede. J. Phys. Chem. B 2006;110:22853-22860. [PubMed: 17092037]

33. Heikal AA, Hess ST, Baird GS, Tsien RY, Webb WW. Molecular spectroscopy and dynamics of intrinsically fluorescent proteins: Coral red (dsRed) and yellow (Citrine). Proc. Natl. Acad. Sci. U.S.A 2000;97:11996-12001. [PubMed: 11050231]

34. Wu JQ, Pollard TD. Counting cytokinesis proteins globally and locally in fission yeast. Science 2005;310:310-314. [PubMed: 16224022]

35. Newman JR, Ghaemmaghami S, Ihmels J, Breslow DK, Noble M, DeRisi JL, Weissman JS. Singlecell proteomic analysis of S. cerevisiae reveals the architecture of biological noise. Nature 2006;441:840-846. [PubMed: 16699522]

36. Yu J, Xiao J, Ren X, Lao K, Xie XS. Probing gene expression in live cells, one protein molecule at a time. Science 2006;311:1600-1603. [PubMed: 16543458]

37. Cai L, Friedman N, Xie XS. Stochastic protein expression in individual cells at the single molecule level. Nature 2006;440:358-362. [PubMed: 16541077]

38. Huang B, Wu H, Bhaya D, Grossman A, Granier S, Kobilka BK, Zare RN. Counting low-copy number proteins in a single cell. Science 2007;315:81-84. [PubMed: 17204646]

39. Periasamy A, Skoglund P, Noakes C, Keller R. An evaluation of two-photon excitation versus confocal and digital deconvolution fluorescence microscopy imaging in Xenopus morphogenesis. Microsc. Res. Tech 1999;47:172-181. [PubMed: 10544332]

40. Rekas A, Alattia JR, Nagai T, Miyawaki A, Ikura M. Crystal structure of Venus, a yellow fluorescent protein with improved maturation and reduced environmental sensitivity. J. Biol. Chem 2002;277:50573-50578. [PubMed: 12370172] 
41. Brejc K, Sixma TK, Kitts PA, Kain SR, Tsien RY, Ormo M, Remington SJ. Structural basis for dual excitation and photoisomerization of the Aequorea victoria green fluorescent protein. Proc. Natl. Acad. Sci. U.S.A 1997;94:2306-2311. [PubMed: 9122190]

42. Battistutta R, Negro A, Zanotti G. Crystal structure and refolding properties of the mutant F99S/ M153T/V163A of the green fluorescent protein. Proteins 2000;41:429-437. [PubMed: 11056031]

43. Usman A, Mohammed OF, Nibbering ETJ, Dong J, Solntsev KM, Tolbert LM. Excited-state structure determination of the green fluorescent protein chromophore. J. Am. Chem. Soc 2005;127:1121411215. [PubMed: 16089429]

44. Hink MA, Visser NV, Borst JW, van Hoek A, Visser AJWG. Practical use of corrected fluorescence excitation and emission spectra of fluorescent proteins in Forster resonance energy transfer (FRET) studies. J. Fluoresc 2003;13:185-188.

45. Moog RS, Kuki A, Fayer MD, Boxer SG. Excitation transport and trapping in a synthetic chlorophyllide substituted hemoglobin: orientation of the chlorophyll S1 transition dipole. Biochemistry 1984;23:1564-1571. [PubMed: 6722108]

46. Wachter RM, Yarbrough D, Kallio K, Remington SJ. Crystallographic and energetic analysis of binding of selected anions to the yellow variants of green fluorescent protein. J. Mol. Biol 2000;301:157-171. [PubMed: 10926499]

\section{Appendix I: Analysis of Dissociation Constant}

In order to obtain the YFP dissociation constant $K_{\mathrm{d}}$ from the concentration dependence of excitation spectra (Fig. 2C), we analyzed the contributions of the various components of our system, which involves two oligomerization states of the protein and two protonation states of the chromophore. Combinations of these two properties result in the multiple species present in YFP at pH 6.0 as listed below

\begin{tabular}{ll}
\hline Name & Definition \\
\hline$m Y F P_{A}$ & monomeric YFP with a neutral chromophore \\
$m Y F P_{B}$ & monomeric YFP with an anionic chromophore \\
$d Y F P_{A A}$ & dimeric YFP with two neutral chromophores \\
$d Y F P_{B B}$ & dimeric YFP with two anionic chromophores \\
$d Y F P_{A B}$ & dimeric YFP with a neutral and an anionic chromophore \\
\hline
\end{tabular}

Assuming a monomer-dimer equilibrium independent of the protonation states of YFP chromophore involved, the dissociation constant can be expressed as

$$
K_{d}=\frac{[m Y F P]^{2}}{[d Y F P]},
$$

where $[m Y F P]$ and $[d Y F P]$ are the total monomer and dimer concentrations, respectively. Letting the total YFP concentration be $c$, it is straightforward to obtain from Eq. A.1 the following dimensionless concentrations

$$
\begin{gathered}
m=\frac{[m Y F P]}{c}=\frac{-\frac{K_{d}}{c}+\sqrt{\left(\frac{K_{d}}{c}\right)^{2}+8 \frac{K_{d}}{c}}}{4}, \\
d=\frac{[d Y F P]}{c}=\frac{1}{2}+\frac{\frac{K_{d}}{c}-\sqrt{\left(\frac{K_{d}}{c}\right)^{2}+8 \frac{K_{d}}{c}}}{8} .
\end{gathered}
$$

For either the monomer or dimer, the ratio of neutral to anionic chromophore is subject to the corresponding acid-base equilibrium. Letting the fraction of neutral form be $A_{m}$ and $A_{d}$ for the monomer and dimer, respectively, the concentrations of the species in the mixture are

$$
\left[m Y F P_{A}\right]=[m Y F P] \times A_{m},
$$




$$
\begin{array}{cc}
{\left[m Y F P_{B}\right]=[m Y F P] \times\left(1-A_{m}\right),} & \text { Eq. A.5 } \\
{\left[d Y F P_{A A}\right]=[d Y F P] \times A_{d}^{2},} & \text { Eq. A.6 } \\
{\left[d Y F P_{B B}\right]=[d Y F P] \times\left(1-A_{d}\right)^{2},} & \text { Eq. A. } 7 \\
{\left[d Y F P_{A B}\right]=[d Y F P] \times 2 A_{d}\left(1-A_{d}\right) .} & \text { Eq. A.8 }
\end{array}
$$

For the yellow fluorescence at $527 \mathrm{~nm}$, the excitation efficiency at $450 \mathrm{~nm}$ is proportional to the total concentration of anionic form including $m Y F P_{B}, d Y F P_{B B}$, and $d Y F P_{A B}$, while the efficiency at $394 \mathrm{~nm}$ is only proportional to $d Y F P_{A B}$ 's concentration due to the hetero-FRET. Therefore, the ratio of these two efficiencies is

$$
\begin{aligned}
& \frac{E_{394}}{E_{450}}=f \times \frac{\left[d Y F P_{A B}\right]}{\left[m Y F P_{B}\right]+\left[d Y F P_{B B}\right] \times 2+\left[d Y F P_{A B}\right]} \\
& =f \times \frac{d \times 2 A_{d}\left(1-A_{d}\right)}{m \times\left(1-A_{m}\right)+d \times 2\left(1-A_{d}\right)},
\end{aligned}
$$

where $m$ and $d$ can be substituted by the expressions in Eqs. A.2 and A.3 and $f$ is a scaling factor. The ratio in Eq. A.9 can be compared with the one calculated from excitation spectra (Fig. 2C), and its concentration dependence is of interest here.

\section{Concentration Dependence and $\mathbf{K}_{\mathbf{d}}$ Estimation}

Using Eq. A.9, we analyze several scenarios that are relevant to the estimation $K_{\mathrm{d}}$. At a concentration that is extremely high, $\lim _{c \rightarrow \infty} m=0$ and $\lim _{c \rightarrow \infty} d=\frac{1}{2}$. Here the ratio in Eq. A.9 becomes $\lim _{c \rightarrow \infty} \frac{E_{394}}{E_{450}}=f \times \frac{\frac{1}{2} \times 2 A_{d}\left(1-A_{d}\right)}{\frac{1}{2} \times 2\left(1-A_{d}\right)}=f \times A_{d}$.

At an intermediate concentration where $c=K_{d}, m=\frac{1}{2}$ and $d=\frac{1}{4}$. From Eq. A.9, we obtain

$$
\begin{aligned}
& \frac{E_{394}}{E_{450}}=f \times \frac{A_{d}\left(1-A_{d}\right)}{\left(1-A_{m}\right)+\left(1-A_{d}\right)} \\
& =\lim _{c \rightarrow \infty} \frac{E_{394}}{E_{450}} \times \frac{1-A_{d}}{\left(1-A_{m}\right)+\left(1-A_{d}\right) .}
\end{aligned}
$$

Therefore, $K_{\mathrm{d}}$ should equal the YFP concentration, where $E_{394} / E_{450}$ is equal to the value at infinite concentration multiplied by a scaling factor, which is a function of only two parameters, $A_{m}$ and $A_{d}$. As a first-order approximation, $A_{m}=A_{d}$ is assumed and this gives

$$
\left.\frac{E_{394}}{E_{450}}\right|_{c=K_{d}}=\lim _{c \rightarrow \infty} \frac{E_{394}}{E_{450}} \times \frac{1}{2} \text {, }
$$

and the excitation spectra in Fig. $2 \mathrm{C}$ would suggest a value of $\sim 9 \mu \mathrm{M}$.

Note the analysis above is based on the assumption that $A_{m}=A_{d}$, that is, the oligomerization state does not affect the ratio of the two protonation states of YFP chromophore. This assumption simplifies the estimation for $K_{\mathrm{d}}$, but is not rigorously correct. This is clearly reflected by the concentration dependence of absorption spectra as shown in Fig. 2D, where it can be seen that $A_{m}>A_{d}$. Since the difference is noticeable, we take this effect into account and estimate a slightly larger value of $10 \sim 15 \mu \mathrm{M}$ for $K_{\mathrm{d}}$.

It should be cautioned that the analysis performed above is based on a few assumptions: $(i)$ The monomer-dimer equilibrium is not affected by the protonation states of YFP chromophore involved; (ii) Different forms of the anionic state, i.e. $m Y F P_{B}, d Y F P_{B B}$, and $d Y F P_{A B}$, contribute 
the same to the yellow fluorescence if they are at the same effective concentration; (iii) The scaling factor $f$ in Eq. A.9 is independent of YFP concentration. If one or more of these assumptions are not valid, the estimate of YFP dimer dissociation constant may deviate from the true value.

\section{Appendix II: Analysis of Transition Moment Orientation Visible Pump/IR Probe Polarization Dichroism of GFP}

Using the coordinates in GFP crystal structures, we calculated the angle $\theta_{\mathrm{C}=\mathrm{O}(\mathrm{Cro})}$ between the bond vector $\mathrm{C} 2=\mathrm{O} 2$ (Fig. 6) and the electronic transition dipole as a function of $\alpha$ (Fig. 7A, solid lines), in comparison with the experimental result of $67 \pm 4^{\circ}$ (26) (Fig. 7A, dashed line). Similar experimental values have been found for the corresponding carbonyl mode in HBDI $\left(62 \pm 4^{\circ}(26) ; 68 \pm 3^{\circ}\right.$ or $\left.66 \pm 3^{\circ}(43)\right)$. Based on this value, $\alpha$ is found to be $8 \pm 3^{\circ}$ or $54 \pm 3^{\circ}$ (the points where the solid lines cross the dashed line). Note that for this calculation, multiple structures of GFP (wild type: 1EMB (41), 1GFL (5) with chains A and B, and 1W7S (24) with chains A, B, C and D; F99S/M153T/V163A GFP (42): 1B9C with chains A, B, C and D) are surveyed to avoid the potential bias that may be associated with individual structures and also to provide an estimation of standard deviation in the calculated angles. As the relatively small error in $\alpha$ reflects, these GFP structures are very similar for the chromophore.

A similar calculation is performed for the angle $\theta_{\mathrm{C}=\mathrm{O}(222)}$ between the bond vector $\mathrm{CD}=\mathrm{OE} 1$ (the name $\mathrm{OE} 1$ is used here to simplify the discussion; the corresponding atoms are labeled as $\mathrm{OE} 2$ in 1B9C for chains $\mathrm{A}$ and $\mathrm{B}$ ) and the electronic transition dipole as a function of $\alpha$ (Fig. 7B, solid lines), in comparison with the experimental result of $28 \pm 4^{\circ}$ (26) (Fig. 7B, dashed line). Note that the vector $\mathrm{CD}=\mathrm{OE} 1$ is calculated from the crystal structures coordinates of GFP in the electronic ground state, while the transient IR experiments measure the angle between the electronic absorption dipole and the transient $\mathrm{C}=\mathrm{O}(222)$ mode in the electronic excited state. With the assumption that no major change occurs in the orientation of $\mathrm{CD}=\mathrm{OE} 1$ following optical excitation of the chromophore and subsequent ESPT to E222, we consider the ground-state structure a reasonable model for that of the excited state. Given the experimental value mentioned above, $\alpha$ is found to be $5 \pm 9^{\circ}$ or $-42 \pm 5^{\circ}$ from all the GFP structures surveyed (Fig. 7B). The relatively large uncertainty in $\alpha$ reflects the structural disorder of the E222 side chain, which has been noticed previously $(24,25)$.

By comparing the two sets of possible $\alpha$ values provided above by the analyses for $\theta_{\mathrm{C}=\mathrm{O}(\mathrm{Cro})}$ and $\theta_{\mathrm{C}=\mathrm{O}(222)}$, only $8 \pm 3^{\circ}$ and $5 \pm 9^{\circ}$ are statistically indistinguishable and can satisfy both experimental observations of the corresponding vibrational dichroism. Therefore, we take the average, $6.5 \pm 5^{\circ}$, as the value of $\alpha$ defining the absorption moment orientation in the plane of the neutral GFP chromophore (Fig. 6).

\section{Correction: Analysis of Absorption Dichroism in GFP Single Crystals (28)}

Previously, polarized absorption spectra of orthorhombic GFP crystals with $P_{212121}$ symmetry were measured to obtain the direction of electronic absorption moment of the chromophore relative to the molecular axes of the protein (28). However, the trigonometric analysis of the absorption dichroism data presented there contains several mistakes, which are corrected below. In addition, because of the space group of the crystals, these dichroism measurements on single crystals generate multiple projections of the transition dipole moment along the principal dichroic axes of the crystal, in contrast to the analysis presented above of the visible pump/IR probe dichroism data which is naturally evaluated in the molecular axis system.

In the orthogonal coordinate system $(\chi, \psi, z)$ constructed by Rosell and Boxer (28), the $z$ axis is defined as the normal to the chromophore plane and the $\chi$ axis is defined as the intersection 
of the chromophore plane and the crystallographic ac plane. With the direction cosines of $\chi$ along the crystallographic axes $(a, b, c)$ defined as

$$
z_{a} \equiv \widehat{e}_{a} \cdot \widehat{z} \quad z_{b} \equiv \widehat{e}_{b} \cdot \widehat{z} \quad z_{c} \equiv \widehat{e}_{c} \cdot \widehat{z},
$$

we obtain the rest of the direction cosines

$$
\begin{gathered}
\widehat{e}_{a} \cdot \widehat{\chi}=-\frac{z_{c}}{\sqrt{1-z_{b}^{2}}} \quad \widehat{e}_{b} \cdot \widehat{\chi}=0 \quad \widehat{e}_{c} \cdot \widehat{\chi}=\frac{z_{a}}{\sqrt{1-z_{b}^{2}}}, \\
\widehat{e}_{a} \cdot \widehat{\psi}=\frac{z_{a} z_{b}}{\sqrt{1-z_{b}^{2}}} \quad \widehat{e_{b}} \cdot \widehat{\psi}=-\sqrt{1-z_{b}^{2}} \quad \widehat{e_{c}} \cdot \widehat{\psi}=\frac{z_{b} z_{c}}{\sqrt{1-z_{b}^{2}}} .
\end{gathered}
$$

Note that none of the expressions in Eq. 3 in Ref. 28 is correct. For instance, since the $\chi$ axis is in the $a c$ plane, the direction cosine of $\chi$ along $b$ should equal zero as shown in Eq. A.14, instead of a non-zero value. In addition, as shown in Eq. A.14 the direction cosine of $\chi$ along $c$ should not equal zero but was taken incorrectly as zero.

These mistakes in direction cosines further propagated into the expressions of absorption dichroism in Eqs. 4-6 of Ref. 28. With the simplifying assumption that the transition moment of GFP lies in the chromophore plane, at some angle $\theta$ relative to the $\chi$ axis, the normalized transition moment vector can be expressed as

$$
\vec{M}=\widehat{\chi} \cos \theta+\widehat{\psi} \sin \theta \text {. }
$$

The absorption dichroism is then expressed as a function of $\theta$

$$
\begin{aligned}
& \frac{O D_{c}}{O D_{a}}=\left(\frac{z_{a} \cot \theta+z_{b} z_{c}}{z_{c} \cot \theta-z_{a} z_{b}}\right)^{2}, \\
& \frac{O D_{c}}{O D_{b}}=\left(\frac{z_{a} \cot \theta+z_{b} z_{c}}{1-z_{b}^{2}}\right)^{2}, \\
& \frac{O D_{a}}{O D_{b}}=\left(\frac{z_{c} \cot \theta-z_{a} z_{b}}{1-z_{b}^{2}}\right)^{2} .
\end{aligned}
$$

The number of distinct solutions for the transition moment orientation relative to the molecular axes yielded by this analysis was eight for each protonation state prior to further scrutiny. Note that half of these solutions are not genuine, because for each value of the $\theta$ angle, two directions in the chromophore plane that are symmetric about the $\chi$ axis are allowed. In addition, the corrected trigonometric analysis is still not straightforward: the direction of the $\chi$ axis needs to be mapped first; the direction of transition moment defined by the $\theta$ angle is then converted to that defined by the $\alpha$ angle in Fig. 6. It is thus desirable to simplify this essentially two-step approach into a more straightforward, one-step approach, in which the absorption dichroism shown in Eqs. A.17-19 is calculated as a function of $\alpha$ directly (Fig. S4). The two-fold redundancy in the number of solutions mentioned above is also avoided.

Based on the crystal structure of wild-type GFP (PDB: 1EMB (41)), the $\alpha$ angle of the neutral chromophore consistent with the data is found to be $1.5,63,-21$, or $76^{\circ}$ (the points where the solid lines cross the dashed line in Fig. S4). By comparing these values with the one obtained above from the vibrational dichroism analysis, $\alpha=6.5 \pm 5^{\circ}$, only $\alpha=1.5^{\circ}$ is statistically indistinguishable from the latter value. 


\section{Appendix III: Analysis of Hetero-FRET Rate Constant}

The orientation factor $\kappa^{2}$ in Eq. 6 is expressed as

$$
\kappa^{2}=\left(\cos \theta_{\text {FRET }}-3 \cos \theta_{1} \cos \theta_{2}\right)^{2},
$$

where $\theta_{F R E T}$ is the angle between the emission dipole of the donor and the absorption dipole of the acceptor, $\theta_{1}$ and $\theta_{2}$ are the angles between these dipoles and the vector connecting the donor and the acceptor (7). The overlap integral $J_{\lambda}$ is given by

$$
J_{\lambda}=\frac{\int_{0}^{\infty} f_{1}(\lambda) \varepsilon_{2}(\lambda) \lambda^{4} d \lambda}{\int_{0}^{\infty} f_{1}(\lambda) d \lambda},
$$

Eq. A.21

where $f_{1}(\lambda)$ is the corrected fluorescence spectrum of the donor, and $\varepsilon_{2}(\lambda)$ is the molar extinction coefficient of the acceptor at wavelength $\lambda$. With $\lambda$ expressed in $\mathrm{nm}$ and $\varepsilon_{2}(\lambda)$ in $\mathrm{M}^{-1} \mathrm{~cm}^{-1}$, Eq. 6 becomes ( 7 )

$$
R_{0}^{6}=8.79 \times 10^{-5} \times \frac{\kappa^{2} \Phi_{1 *} J_{\lambda}}{n^{4}}
$$

in $\AA^{6}$. Substituting Eq. A.22 into Eq. 5 yields

$$
k_{\mathrm{FRET}}^{\text {hetero }}=8.79 \times 10^{-5} \times \frac{\kappa^{2} J_{\lambda}}{R^{6} n^{4}} k_{r, 1},
$$

where $k_{r, 1}$ is the radiative decay rate of the neutral chromophore, which can be approximated by that of the anionic chromophore $k_{r, 2}$ (see Scheme 1), $1.9 \times 10^{8} \mathrm{~s}^{-1}$, calculated previously for YFP 10C (6).

To estimate the angles $\theta_{1}$ and $\theta_{2}$ in Eq. A.20 and the distance $R$ in Eq. A.23, we assume that the interchromophore vector $1 \rightarrow 2$ connects the midpoint of the vector CG2 $\rightarrow$ CA2 in the donor and that in the acceptor. With the same set of assumptions as made above for anisotropy, we calculated the orientation factor $\kappa^{2}$ as a function of angle $\alpha$ (Fig. 7D, green curve). For $\alpha=6.5$ $\pm 5^{\circ}, \kappa^{2}=2.6 \pm 0.3$. In the other two scenarios discussed for anisotropy with the transition dipole of the anionic acceptor chromophore rotated away from this value clockwise or counterclockwise by $15^{\circ}$, we found $\kappa^{2}=2.0 \pm 0.3$ or $2.9 \pm 0.3$, respectively (Fig. 7D, red and blue curves). In addition, the length of the interchromophore vector $1 \rightarrow 2$ is calculated to be $25.5 \AA$ Á. An alternative approach to estimating the distance $R$ is to calculate all the 169 (13 by 13) separations between any heavy atom that is part of the conjugation (see Fig. 1D) in the donor and another such atom in the acceptor. This approach yields an interchromophore distance of $24.3 \pm 2.9 \AA$, consistent with the value above.

To calculate the overlap integral $J_{\lambda}$, it would be desirable to use the fluorescence spectrum of the neutral chromophore in YFP 10C; however, this is difficult to obtain due to the lack of appreciable blue fluorescence from the neutral chromophore under normal conditions. Using the fluorescence spectrum of a T203F GFP mutant that exhibits an emission peak at $\sim 460 \mathrm{~nm}$ (not shown), we obtained a value of $1.0 \times 10^{15} \mathrm{M}^{-1} \mathrm{~cm}^{-1} \mathrm{~nm}^{4}$ for $J_{\lambda}$; however, there exists uncertainty in the red region of the spectrum due to the overlapping emission from the anionic chromophore. In the presence of thiocyanate, YFP 10C itself exhibits strong blue fluorescence with a peak at $\sim 480 \mathrm{~nm}$ (not shown), which yields a value of $1.5 \times 10^{15} \mathrm{M}^{-1} \mathrm{~cm}^{-1} \mathrm{~nm}^{4}$ for $J_{\lambda}$. This likely provides an upper limit, because the spectrum is red-shifted from the one observed in the absence of thiocyanate. Therefore, an intermediate value of $1.25 \times 10^{15}$ $\mathrm{M}^{-1} \mathrm{~cm}^{-1} \mathrm{~nm}^{4}$ is used instead, which is comparable to the one for the EBFP-EGFP pair (44). This value is considered to approximate that for the $\mathrm{YFPH}_{\mathrm{D}^{-}} \mathrm{YFP}_{\mathrm{A}}{ }^{-}$pair (Scheme 1), given the spectroscopic similarity between the two pairs and the fact that the $J_{\lambda}$ for $\mathrm{YFPH}_{\mathrm{D}^{-}}$ $\mathrm{YFP}_{\mathrm{A}}{ }^{-}$should be smaller than the value for the ECFP-EYFP pair (44), $1.55 \times$ 
$10^{15} \mathrm{M}^{-1} \mathrm{~cm}^{-1} \mathrm{~nm}^{4}$, where the spectral overlap between the donor emission and acceptor absorption is better.

With the parameters obtained above and a refractive index of 1.33 (45), we calculated the rate constant for FRET in YFP hetero-dimer, $k_{\mathrm{FRET}}^{\text {hetero }}=(8.4 \pm 3.0) \times 10^{10} \mathrm{~s}^{-1}$. Note that only the largest source of error, the interchromophore distance $R$, was considered for the estimation of error in $k_{\mathrm{FRET}}^{\text {hetero }}$, as a result of the sixth power dependence in Eq. 5. A 5\% error, e.g. $1.2 \AA$, was assumed for $R$, which is not well defined.

\section{Materials and Methods}

\section{Sample Preparation}

Recombinant H148G YFP and YFP 10C were expressed and purified as described previously (3). Mutagenesis (A206K) was performed using the QuikChange kit (Stratagene, La Jolla, CA) on the background of the YFP 10C construct previously used (6). Proteins were exchanged into a buffer solution by concentrating the sample to a minimum volume, followed by resuspension in the desired buffer, and repeating this process three times. Buffer solutions were prepared with $40 \mathrm{mM} \mathrm{NaCl}, 1 \mathrm{mM} \mathrm{MgCl}$, and $20 \mathrm{mM}$ buffer (Citric acid, MES, MOPS, HEPES, TAPS or Bis-tris propane as appropriate). A pH 6.0 buffer with the components above, i.e. in MES, plus $400 \mathrm{mM} \mathrm{Na}_{2} \mathrm{SO}_{4}$ was prepared to examine the effect of ionic strength. Here $\mathrm{Na}_{2} \mathrm{SO}_{4}$ was chosen over $\mathrm{NaCl}$ as the reagent for increasing the ionic strength, due to the known effect of halide binding to the YFP chromophore and the lack of such an effect for sulfate (46).

\section{Absorption and Steady-State Fluorescence}

Absorption spectra were collected with a Cary 6000i spectrophotometer (Varian, Palo Alto, CA) using a 1-mm pathlength quartz cuvette. Corrected excitation spectra for fluorescence at $527 \mathrm{~nm}$ were collected with the same cuvette using front-face geometry on a Spex FluoroLog-3 spectrofluorimeter (HORIBA Jobin Yvon, Edison, NJ) equipped with a cooled PMT detector. The spectra collected with the buffer only were subtracted out. Steady-state fluorescence anisotropy at $527 \mathrm{~nm}$ with $400 \mathrm{~nm}$ excitation was measured using a 10-mm pathlength quartz cuvette with right-angle geometry. The signal of buffer only was subtracted out. The proper G-factor was determined and applied to anisotropy calculation.

\section{YFP 10C Concentration}

YFP 10C's molar extinction coefficient at $280 \mathrm{~nm}, 21800 \mathrm{M}^{-1} \mathrm{~cm}^{-1}$, was obtained by measuring the absorption spectrum at $\mathrm{pH} 9.2$, and dividing the absorbance at $280 \mathrm{~nm}$ by that at $514 \mathrm{~nm}$, multiplied by the published molar extinction coefficient of YFP at $514 \mathrm{~nm}, 83400$ $\mathrm{M}^{-1} \mathrm{~cm}^{-1}$. YFP concentration was calculated using this molar extinction coefficient at $280 \mathrm{~nm}$, except in dilution experiments where the dilution factors and the concentration of the stock solution were used for calculation.

\section{Time-Resolved Fluorescence}

Time-resolved fluorescence was measured for YFP and H148G YFP at $527 \mathrm{~nm}$, using a timecorrelated single photon counting setup equipped with an R3809U-50 MCP detector (Hamamatsu, Japan) and an SPC-630 module (Becker \& Hickl, Germany) as described previously (6). In brief, samples were excited by $400 \mathrm{~nm}$ pulses generated from the second harmonic of an argon-ion laser pumped titanium-sapphire laser operating at $82 \mathrm{MHz}$ (Spectra Physics, Mountain View, CA). The instrument response function measured with scattered excitation light has a typical FWHM of 30 ps. The detector was cooled to suppress the dark count rate and black boards were used to block scattered light for the experiments using very 
dilute samples. $1.0 \mathrm{~mW}$ of excitation light was used to illuminate the sample which was rapidly stirred in a quartz cuvette with 1-mm pathlength, and the motorized $\lambda / 2$-plate that controlled the excitation polarization was rotated constantly between the parallel and perpendicular positions, relative to emission with a Glan-Thompson polarizer. Time-resolved anisotropy was obtained by summing the data at each polarization and calculating with the formula in Eq. A. $24(7)$

$$
r(t)=\frac{I_{\|}(t)-I_{\perp}(t)}{I_{\|}(t)+2 I_{\perp}(t)} .
$$

\section{Two-Photon Excitation}

Time-resolved two-photon excited fluorescence was acquired for wild-type GFP and YFP on the same setup for the one-photon experiments; however, the $400 \mathrm{~nm}$ second harmonic of titanium-sapphire pulses was not generated. Instead, the unamplified $800 \mathrm{~nm}$ fundamental pulses with pulse energy of $5.7 \mathrm{~nJ}$ were focused into the sample to induce fluorescence. No detectable photobleaching of the sample was observed under this condition.

\section{Pump-Probe}

Transient absorption spectra were collected for wild-type GFP and YFP, using a pump-probe setup equipped with a titanium-sapphire regenerative amplified source (Spectra Physics, Mountain View, CA) and a CCD detection system (Princeton Instruments, Trenton, NJ). The pump at $390 \mathrm{~nm}$ was generated from the second harmonic of amplified fundamental pulses and was used to excite the sample which was rapidly stirred in a quartz cuvette with 1-mm pathlength. A white-light continuum was generated by focusing the $780 \mathrm{~nm}$ pulses with pulse energy of $4 \mu \mathrm{J}$ into the water in a 1-mm cuvette and this white light was then separated into two beams: one was used as the probe beam which crossed with the pump in the sample and the other was used as a reference beam to correct for the intensity and spectral fluctuations of the continuum generation. These two beams were focused into two separate optical fibers, the outputs of which were connected to a $0.3-\mathrm{m}$ monochromator and a $1340 \times 100$ pixel CCD detector. Difference absorption spectra were obtained with the pump on and off. 

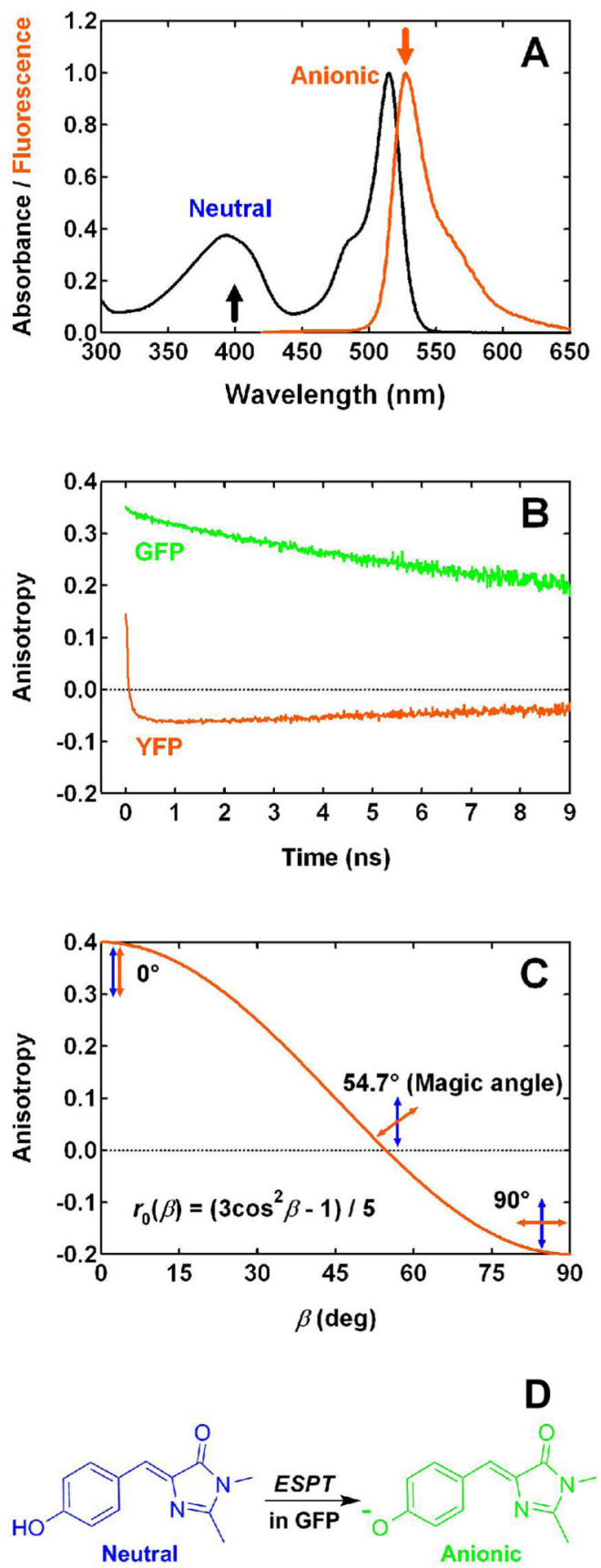

Figure 1.

(A) Normalized absorption and fluorescence $\left(\lambda_{\mathrm{ex}}=400 \mathrm{~nm}\right)$ spectra of YFP 10C at $\mathrm{pH}$ 6.0. Arrows pointing upwards and downwards indicate the excitation and emission wavelengths relevant to anisotropy measurements. Protonation states of the chromophore are annotated for both absorption bands. (B) Time-resolved fluorescence anisotropy measured for wild-type GFP at $\mathrm{pH} 7.8\left(\lambda_{\mathrm{em}}=508 \mathrm{~nm}\right)$ and YFP $10 \mathrm{C}$ at $\mathrm{pH} 6.0\left(\lambda_{\mathrm{em}}=527 \mathrm{~nm}\right)$ with $400 \mathrm{~nm}$ one-photon excitation. (C) Dependence of one-photon excited fluorescence anisotropy on $\beta$, the angle between absorption (blue arrow) and emission (orange arrow) dipole moments. Three different cases are illustrated, with $\beta=0,54.7$ (magic angle), and $90^{\circ}$, respectively. (D) Excited-state 
proton transfer in GFP is illustrated, with the neutral and anionic states of the chromophore shown in blue and green, respectively. 

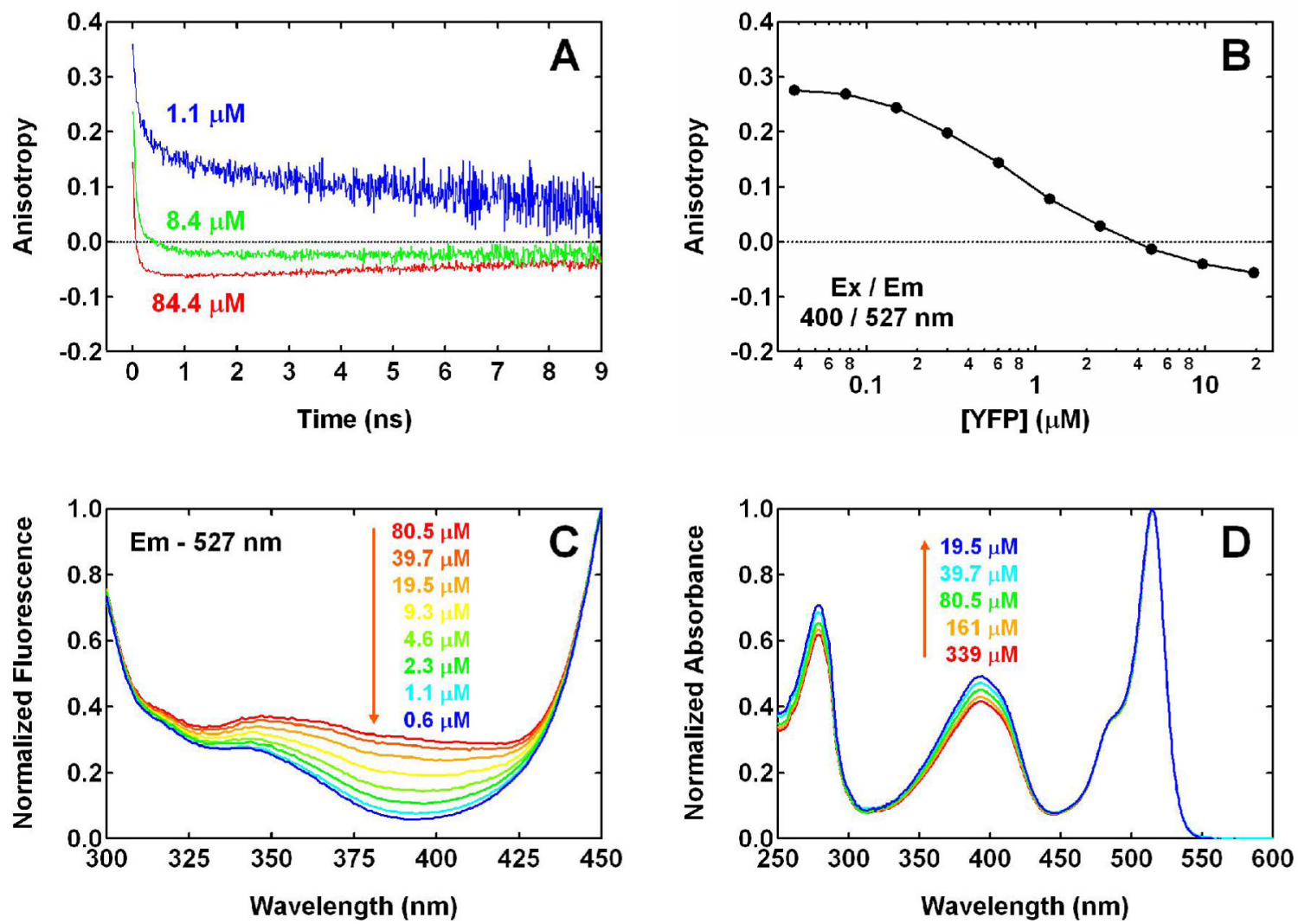

Figure 2.

(A) Time-resolved and (B) steady-state anisotropy of $527 \mathrm{~nm}$ fluorescence, measured for YFP $10 \mathrm{C}$ at $\mathrm{pH} 6.0$ with $400 \mathrm{~nm}$ one-photon excitation. The protein concentrations, except the highest one in both panels, are calculated from the dilution factors. The error bars of steadystate anisotropy, calculated from the standard deviation of 5 measurements at each concentration, are smaller than the size of the markers in panel B and thus not shown. (C) Excitation $\left(\lambda_{\mathrm{em}}=527 \mathrm{~nm}\right)$ and (D) absorption spectra of YFP 10C at pH 6.0 as a function of concentration, which are normalized to the intensity at 450 and $514 \mathrm{~nm}$, respectively. The three lowest concentrations in panel $\mathrm{C}$ are calculated from the corresponding dilution factors. 

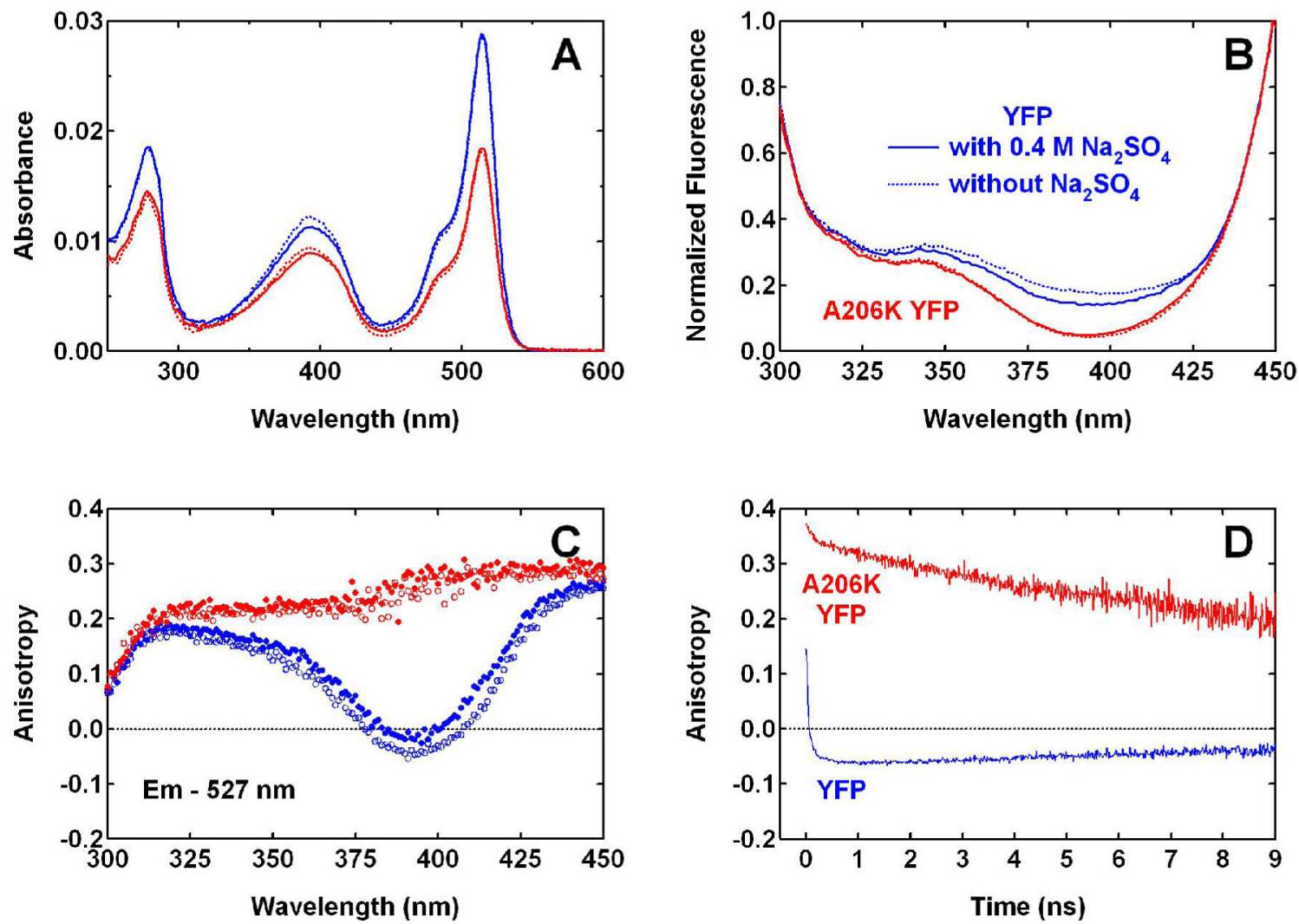

Figure 3.

(A) Absorption and (B) normalized excitation $\left(\lambda_{\mathrm{em}}=527 \mathrm{~nm}\right)$ spectra of YFP 10C (blue) and A206K YFP (red) at pH 6.0. Dashed lines are spectra of proteins in normal pH 6.0 buffer (40 $\mathrm{mM} \mathrm{NaCl}, 1 \mathrm{mM} \mathrm{MgCl}_{2}$, and $20 \mathrm{mM} \mathrm{MES}$ ). Solid lines are spectra of proteins in $\mathrm{pH} 6.0$ buffer with the components above plus $400 \mathrm{mM} \mathrm{Na}_{2} \mathrm{SO}_{4}$, added to increase the ionic strength. (C) The corresponding steady-state anisotropy for $527 \mathrm{~nm}$ fluorescence, with unfilled and filled circles representing the buffer conditions without and with $400 \mathrm{mM} \mathrm{Na}_{2} \mathrm{SO}_{4}$, respectively. (D) Time-resolved anisotropy measured for YFP and A206K YFP at $527 \mathrm{~nm}$ in normal pH 6.0 buffer with $400 \mathrm{~nm}$ one-photon excitation. 

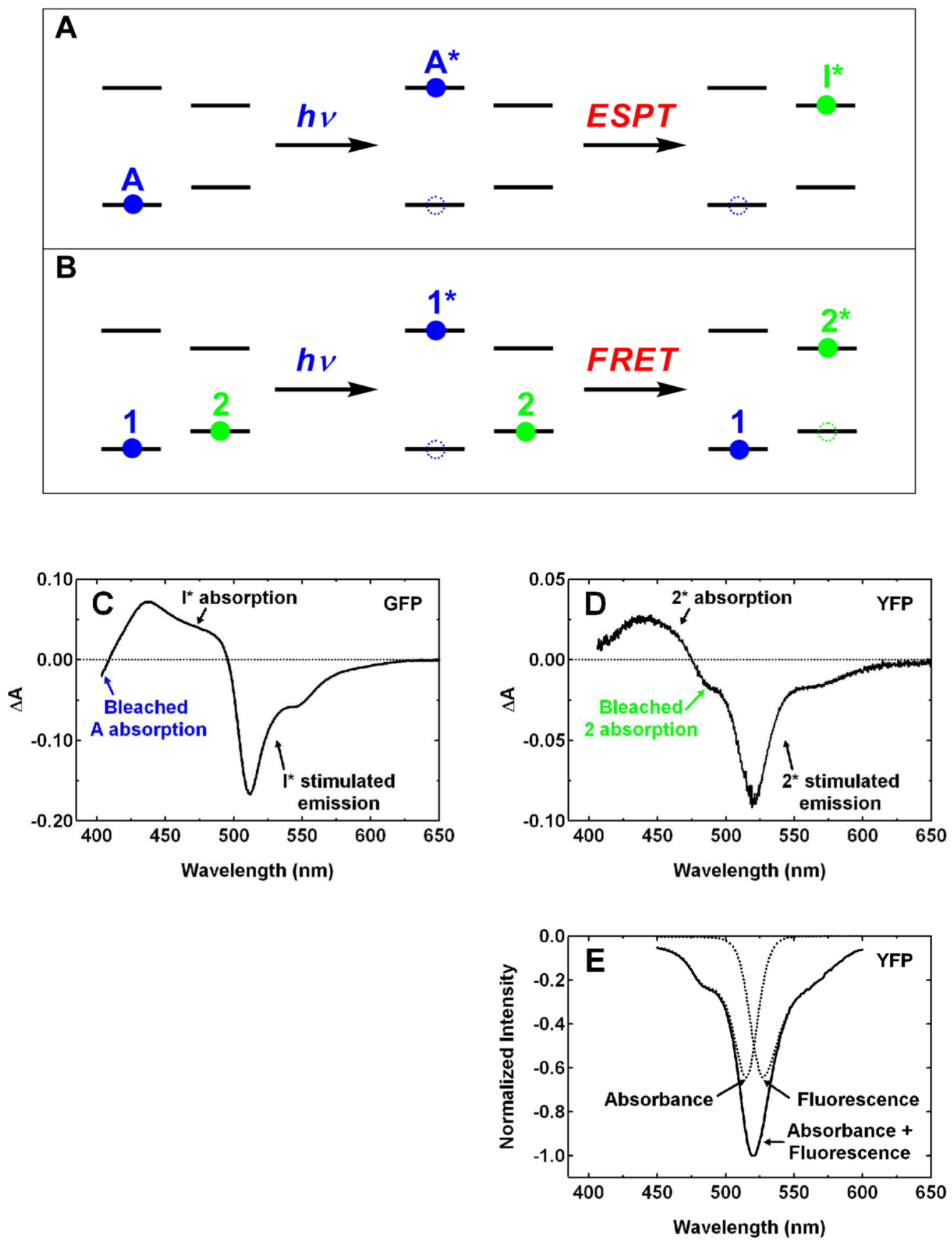

Figure 4.

Illustration of the states and processes involved in the excited-state proton transfer (A) and Förster resonance energy transfer (B). Blue and green circles represent neutral and anionic states, respectively. Filled and unfilled circles represent occupied states and transient absorption bleaches, respectively. Pump-probe spectra at $\sim 450 \mathrm{ps}$ following $390 \mathrm{~nm}$ femtosecond excitation pulses are shown for wild-type GFP at pH 7.8 (C) and YFP 10C at pH 6.0 (D), respectively. The band assignment for GFP by Kennis et al. (23) is followed in panel C. The spectra in $\mathrm{C}$ and $\mathrm{D}$ are consistent with the models shown in the rightmost panels of $\mathrm{A}$ and $\mathrm{B}$, respectively. (E) Illustration of the contributions from the bleached absorption and stimulated emission of the anionic chromophore 2 to the transient absorption signal between 
450 and $600 \mathrm{~nm}$. Steady-state absorption and fluorescence spectra (see Fig. 1A) with equal peak intensity are inverted in sign to model these two contributions (dashed lines), respectively. 


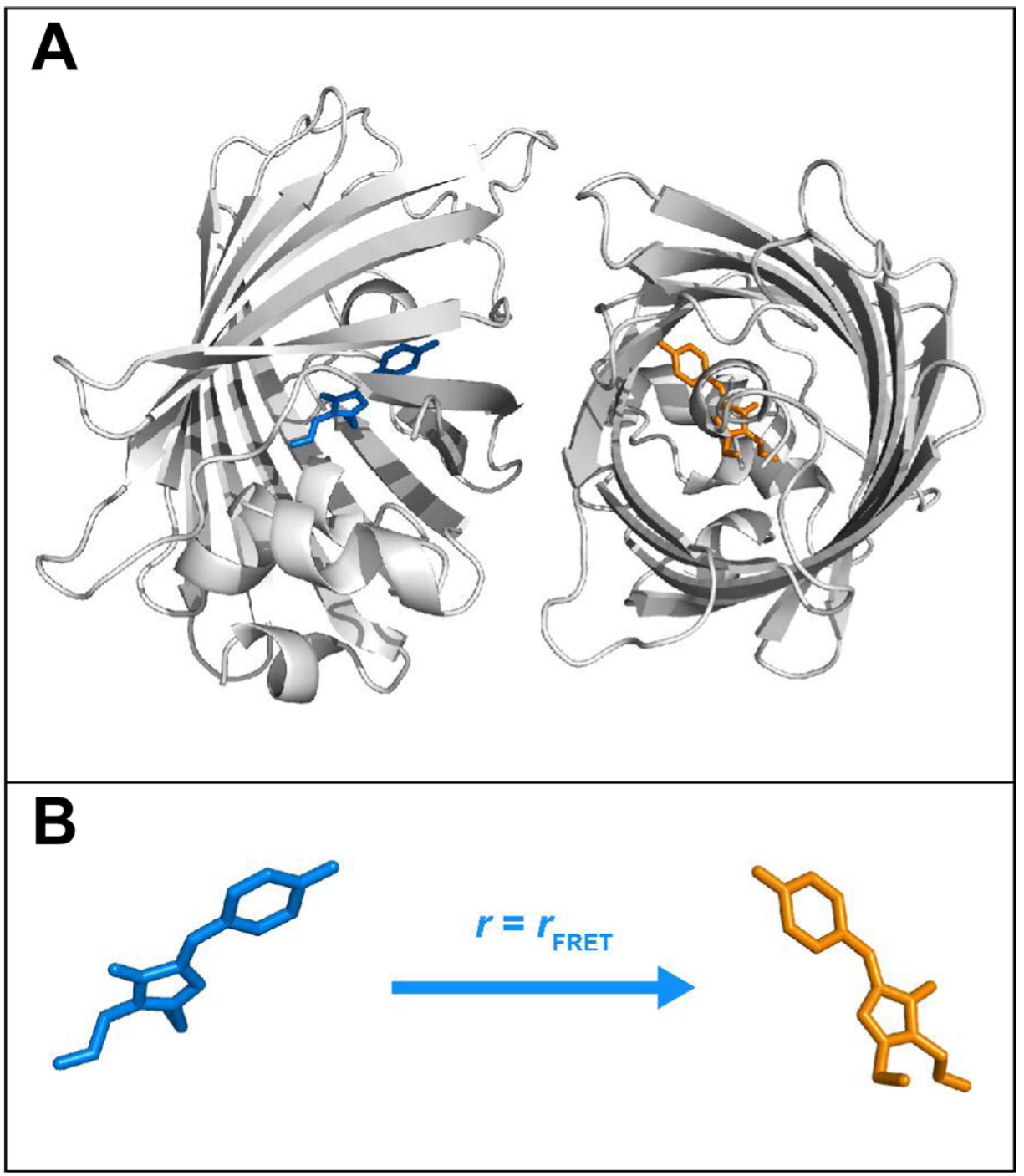

Figure 5.

(A) Structural model of YFP dimer and (B) the corresponding anisotropy of the unidirectional hetero-FRET. Ribbon and stick structures are shown for the protein and the chromophore, respectively. The neutral and anionic chromophores are colored in blue and orange, respectively. The images were created using the coordinates from a crystallographic YFP dimer (PDB code: 1YFP) as follows: one monomer was obtained directly from chain A in the PDB file; for the other monomer, the PyMOL (DeLano Scientific LLC) command, symexp, was executed to generate the symmetry-related object sym02000000, which corresponds to the symmetry operator $(1 / 2+\mathrm{X}, 1 / 2-\mathrm{Y}, 1-\mathrm{Z})$, from chain B in the PDB file; thus, the dimer interface between the two monomers, including residues Ala206, Leu221, and Phe223, which was observed for wild-type GFP (5), YFP 10C (3), and Venus (40) can be visualized (not shown for clarity), while the coordinates of chain B in the PDB file does not produce this interface with chain A. The structure of the YFP dimer in the crystal may not necessarily reflect that in solution, but is a starting point for the quantitative analysis of the hetero-FRET (see text for discussion). 


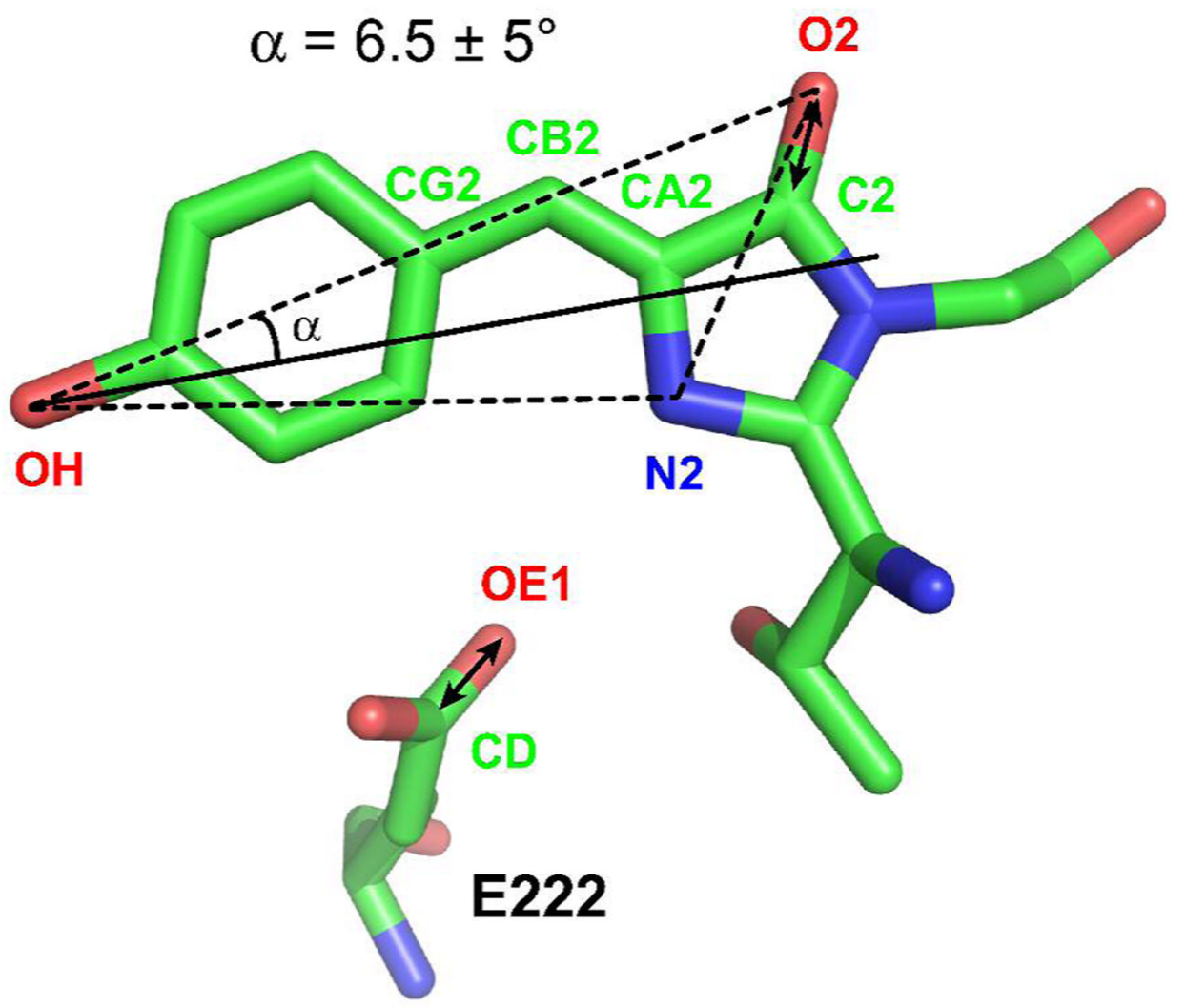

Figure 6.

Crystal structure of the chromophore and residue E222 of wild-type GFP (PDB code: 1EMB (41)). Carbon, oxygen, and nitrogen atoms are colored in green, red, and blue, respectively. Selected atoms are labeled following the nomenclature used in the PDB file. Double-headed arrows represent the vibrational transition dipole of carbonyl stretch in the chromophore and E222. The electronic transition dipole of the chromophore is assumed to be in the plane defined by the 3 noncollinear atoms $\mathrm{OH}, \mathrm{O} 2$ and $\mathrm{N} 2$, rotated from the vector $\mathrm{OH} \rightarrow \mathrm{O} 2$ by an angle $\alpha$ towards the vector $\mathrm{OH} \rightarrow \mathrm{N} 2$. The sign of $\alpha$ corresponds to the direction of rotation, positive for clockwise and negative for counterclockwise. As described in the text and Appendix II, a value of $\alpha=6.5 \pm 5^{\circ}$ is obtained based on the analysis of published visible pump/IR probe polarization dichroism data of GFP (26). 

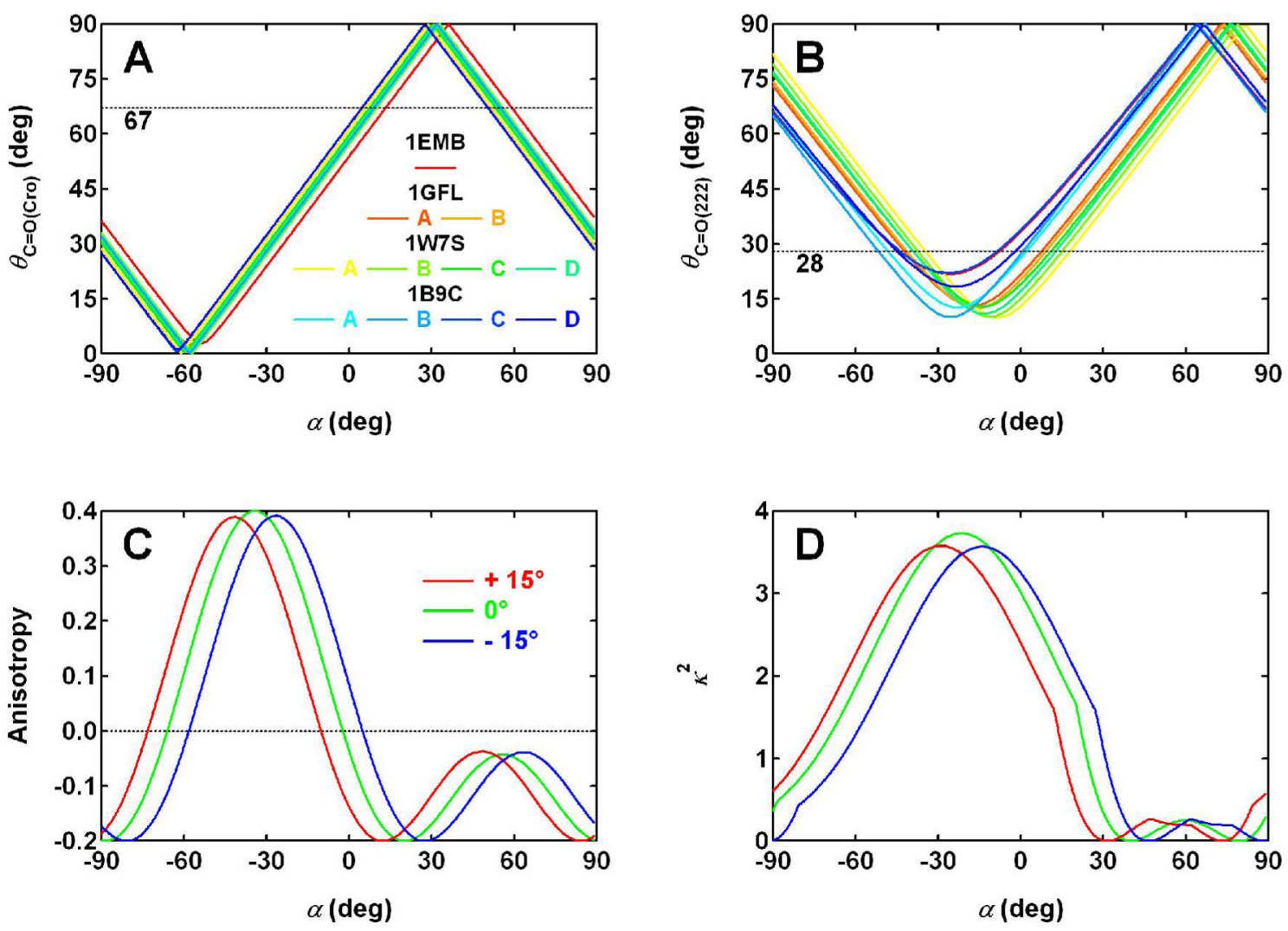

Figure 7.

(A) The angle $\theta_{\mathrm{C}=\mathrm{O}(\mathrm{Cro})}$ between the bond vector $\mathrm{C} 2=\mathrm{O} 2$ (Fig. 6) and the electronic transition dipole calculated as a function of angle $\alpha$ (solid lines), using the coordinates in GFP crystal structures. The experimental value of $\theta_{\mathrm{C}=\mathrm{O}(\mathrm{Cro})}, 67^{\circ}$, measured with polarization-resolved, visible pump/IR probe technique (26) is also shown (dashed line) for comparison. (B) The angle $\theta_{\mathrm{C}=\mathrm{O}(222)}$ between the bond vector $\mathrm{CD}=\mathrm{OE} 1$ (Fig. 6) and the electronic transition dipole as a function of $\alpha$ (solid lines), in comparison with the experimental value of $28 \pm 4^{\circ}$ (26) (dashed line). Multiple structures of GFP (wild type: 1EMB (41), 1GFL (5) with chains A and B, and 1W7S (24) with chains A, B, C and D; F99S/M153T/V163A GFP (42): 1B9C with chains A, B, C and D) are used for the calculations in panels A and B to avoid the potential bias that may be associated with individual structures and also to provide an estimation of standard deviation of the calculated angles. (C) The anisotropy and (D) orientation factor $\kappa^{2}$ associated with the unidirectional FRET in YFP hetero-dimer (Fig. 5, PDB code: 1YFP (3)) calculated as a function of $\alpha$ (green curves). Two other scenarios are also considered, with the transition dipole of the acceptor, anionic chromophore, being rotated away from the orientation specified by $\alpha$ clockwise or counterclockwise by $15^{\circ}$ (red and blue curves, respectively). 


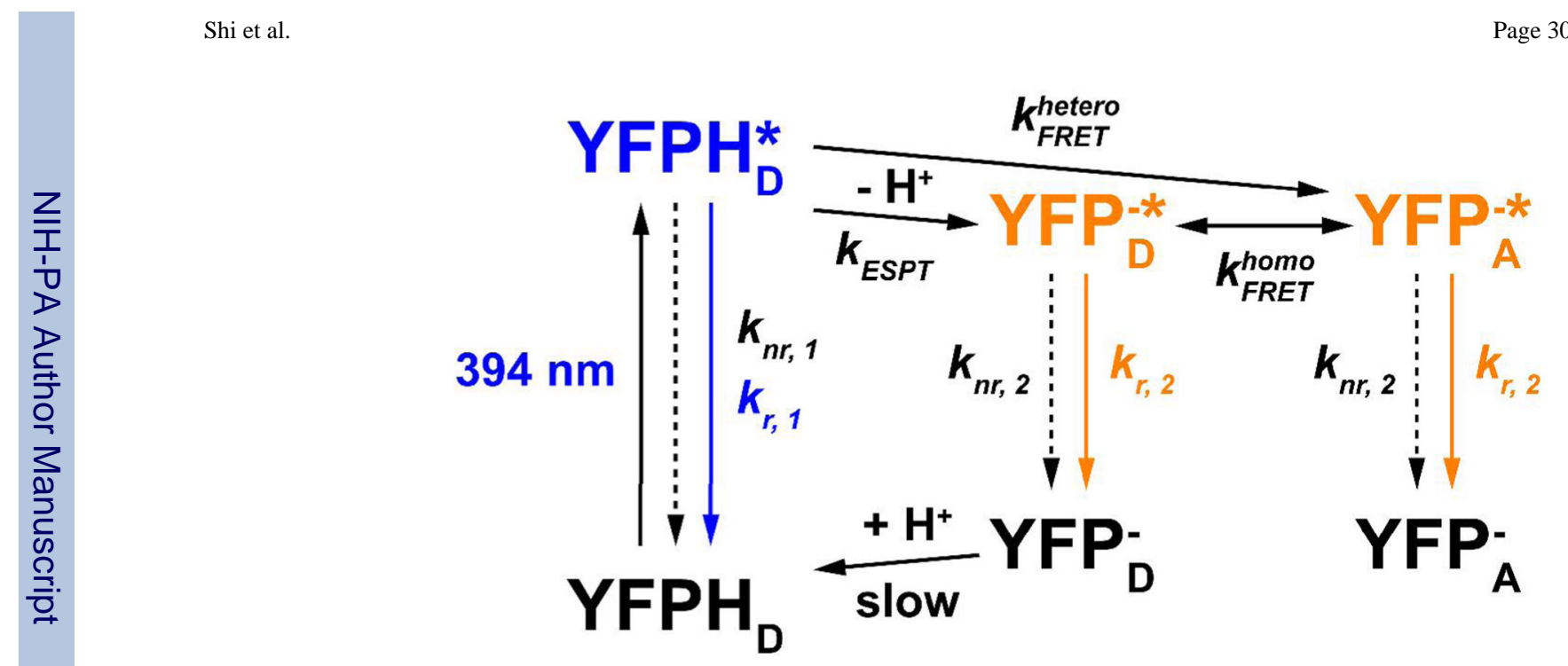

Scheme 1. 